\title{
Modelo de Potts Diluído
}

Fredy Walther Castellares Cáceres

DISSERTAÇÃO APRESENTADA

AO

INSTITUTO DE MATEMÁTICA E ESTATÍSTICA

DA

UNIVERSIDADE DE SÃO PAULO

PARA

OBTENÇÃO DO GRAU DE MESTRE

EM

ESTATÍSTICA

Área de Concentração: PROBABILIDADE

Orientador: Prof. Dr. Luiz Renato Gonçalves Fontes

O autor recebeu auxilio financeiro da FAPESP. Processo 98/05109-9

São Paulo, 24 de julho de 2000 


\section{Modelo de Potts Diluído}

Este exemplar corresponde à redação final da dissertação corrigida e defendida por Fredy Walther Castellares Cáceres

e aprovada pela comissão julgadora.

São Paulo, 24 de julho de 2000.

Banca examinadora:

- Prof. Dr. Luiz R. Fontes (Presidente) - IME-USP

- Prof. Dr. Pablo Ferrari - IME-USP

- Prof. Dr. Henrique von Dreifus- IME-USP 
Dedicado a mis viejos, Lucy y Máximo.

Para mis hermanos, Edwin y César.

Por todo lo que significan para mí. 


\section{Agradecimentos}

Ao Povo Brasileiro representado na FAPESP por permitir-me conhecer seu pais e parte de sua cultura.

A Luiz Renato por apresentar-me um dos modelos mais lindos e interessantes da teoria da Probabilidade e pela dedicada orientação

Ao grupo de Probabilidade pelo coleguismo. Em especial a Vladimir Belitsky.

A Carlos Pantaleón por su ayuda con el Latex.

Finalmente a mis amigos Antonio Pereyra, Guillermo Cuba e Adam Sargeant por su apoyo y fuerza.

Gracias Toño, por todo..... 


\section{Resumo}

O objetivo da dissertação é apresentar o modelo de aglomerados aleatórios e estudar sua relação com o modelo de Potts. Usando o modelo de aglomerados aleatórios, daremos condições necessárias e suficientes para a existência de magnetização espontânea nos modelos de Potts ferromagnético, homogêneo e diluído definido sobre a rede hipercúbica d-dimensional.

Mostramos que existe quase certamente uma temperatura crítica determinística no modelo diluído, que é trivial (zero) se a probabilidade de não-diluição for menor ou igual à probabilidade crítica do modelo de percolação associado aos elos diluídos (percolação independente), e não trivial (positiva finita), caso contrario. Neste último caso, para temperaturas abaixo da crítica, mostramos que para quase toda realização da diluição, todo sítio que estiver num aglomerado infinito de elos não-diluídos está magnetizado. 


\section{Abstract}

The objective of this dissertation is to present the random cluster model and study its relation with the Potts model. Using the random cluster model we give necessary and sufficient conditions for the existence of spontaneous magnetization in the homogeneous and dilute ferromagnetic Potts model defined on the hypercubic d-dimensional lattice.

We show that there almost surely exists a critical deterministic temperature in the dilute model, which is trivial (zero) if the probability of non-dilution is less than or equal to the critical probability of the percolation model associated to the dilute bonds (independent percolation), and non-trivial (positive almost surely and finite), in the opposite case. In the latter case, for temperatures below critical, we show that almost surely site in an infinite cluster of non-dilute bonds is magnetized. 


\section{Índice}

Agradecimentos $\quad$ V

Resumo vi

Abstract vii

1 Introdução 1

2 Modelo de aglomerados aleatórios 4

2.1 Definição do modelo. . . . . . . . . . . . . . . 5

2.2 Medida FKG. . . . . . . . . . . . . . . . . 7

2.3 Desigualdades de comparação. . . . . . . . . . . . . . . 11

2.4 Limite Termodinâmico . . . . . . . . . . . . . . . . . . . . . 13

2.5 Transição de Fase . . . . . . . . . . . . . . . . . . . 16

3 Modelo de Potts 20

3.1 Definição do modelo. . . . . . . . . . . . . . . 20

3.2 Representação FK . . . . . . . . . . . . . . . . . . . . . . 24

3.3 Valores médios no modelo de Potts. . . . . . . . . . . . . . 28

3.4 Limite Termodinâmico para o Modelo de Potts. . . . . . . . . 30

3.5 Transição de Fase no Modelo de Potts. . . . . . . . . . . . . . . 32 
4 Modelo de Potts diluído 34

4.1 Definição do modelo . . . . . . . . . . . . . . . . . 34

4.2 Resultados principais. . . . . . . . . . . . . . . . 36

Referências Bibliográficas $\quad 41$ 


\section{Introdução}

Neste trabalho abordamos alguns modelos da Mecânica Estatística, tais como o modelo de Potts e o modelo de aglomerados aleatórios. Este tipo de modelos é estudado pela Mecânica Estatística de equilíbrio. Esta descreve o comportamento de sistemas físicos com muitos componentes que estão em equilíbrio térmico com seu meio externo ( ver Ellis [6] e Ruelle [18]).

O modelo de aglomerados aleatórios que foi proposto por Fortuin e Kasteleyn [8] apareceu como uma generalização do modelo de percolação. O modelo de percolação que fora formulado por Broadbent e Hamersley [5] nos permite modelar por exemplo o fenômeno de transporte de um fluído através de um meio poroso. O meio é constituído de poros e de canais microscópicos por onde passaria o fluído. Numa situação simples cada canal pode estar aberto ou fechado à passagem do fluído, dependendo de diferentes características que podem ser resumidas num parâmetro. Para nosso interesse cada canal independentemente dos demais estará aberto com probabilidade $p$, o parâmetro do modelo, e estará fechado com probabilidade $1-p$. No modelo de aglomerados aleatórios homogêneos temos que além do parâmetro $p$, existe outro parâmetro que introduz uma certa dependência no modelo. A questão básica de nosso estudo será a ocorrência ou não de percolação, isto é, a existência de um caminho infinito de elos abertos atravessando o meio.

Vamos modelar o meio físico microscópicamente pela geometria do grafo $\left(Z^{d}, E^{d}\right)$ onde o conjunto de sítios do grafo $\left(Z^{d}\right)$ representam os poros e o conjunto de elos 
do grafo $\left(E^{d}\right)$ representam os canais do meio. Nesta geometria o modelo de aglomerados aleatórios descreve um processo de percolação de elos dependentes. Uma das propriedades do modelo é descrever sistemas magnéticos como o modelo de Potts ferromagnético (sistemas ferromagnéticos são aqueles que podem ter uma imantação espontânea sem campo magnético externo). A principal vantagem do modelo de aglomerados é expressar correlações no modelo de Potts como magnitudes percolativas. Por exemplo, a magnetização no modelo de Potts é a probabilidade de percolação no modelo de aglomerados aleatórios.

Descreveremos agora a organização da dissertação: No capítulo dois fizemos um estudo euxautivo do modelo de aglomerados aleatórios definidos sobre o grafo $\left(Z^{d}, E^{d}\right)$. Definimos as medidas de aglomerados aleatórios como medidas limite de convergência fraca das medidas de aglomerados aleatórios definidas sobre um grafo finito. Não usamos para a definição destas medidas as condições DLR (Drobrushin, Lanford e Ruelle) só com a intenção de deixar a exposição da dissertação a nível mais elementar possivel. Como resultados principais apresentamos as desigualdades de comparação para duas medidas de aglomerados aleatórios com diferentes parâmetros. Além disto estudamos o limite termodinâmico e a transição de fase para o modelo. A existência da probabilidade de percolação é demonstrada usando o resultado da existência da probabilidade de percolação do modelo de percolação de elos independentes e as desigualdades de comparação das medidas de aglomerados.

No capítulo três estudamos o modelo de Potts ferromagnético, apresentamos os conhecidos resultados tradicionais usando a representaçào de Fortuin e Kasteleyn (Representação FK), mostrando que o modelo de aglomerados aleatórios é equivalente ao modelo de Potts. Da mesma forma como no capítulo dois aqui apresentamos as medidas de Gibbs correspondentes ao modelo de Potts de $q$ estados sem usar as condições DLR. novamente introduzimos as medidas de Potts como medidas limites de convergência fraca.

Com respeito à transição de fase no modelo de Potts de $q$ estados nós evitamos falar sobre a unicidade das medidas de Gibbs e em todo momento estudamos à magnetização como parâmetro de ordem para investigar a transição de fase. O estudo 
das condições DRL e o estudo da transição de fase em termos da unicidade das medidas de Gibbs escapam ao nível elementar e a intenção da dissertação é apresentar o modelo de aglomerados aleatórios ao nível elementar mas não trivial e estudar com este modelo alguns problemas interessantes dos modelos de Potts.

No capítulo quatro fizemos o estudo do modelo de Potts ferromagnético diluído. O modelo de Potts é dito diluído se a substância em estudo contem elos não-magnéticos entre os elos magnéticos, de modo que só haverá interação entre átomos que estejam ligados entre si por dois elos magnéticos. Neste capítulo apresentamos um dos resultados principais da dissertação. Mostramos que existe quase certamente uma temperatura crítica determinística no modelo diluído, que é trivial (zero) se a probabilidade de não-diluição for menor o igual à probabilidade crítica do modelo de percolação associado aos elos diluídos (percolação independente), e não trivial (positiva finita), caso contrário. Neste último caso, para temperaturas abaixo da crítica, mostramos que para quase toda realização da diluição, todo sítio que estiver num aglomerado infinito de elos não-diluídos está magnetizado.

Para finalizar comentaremos que nos últimos anos a teoria da percolação em particular e os Sistemas de Partículas em geral estão sendo estudados em geometrias mais gerais que um hipercubo ou uma árvore e as técnicas que estão sendo criadas requerem de uma combinação elegante das propriedades geométricas em que se representam os processos e as propriedades matemáticas (para a percolação, exploram-se as propriedades algébricas dos grupos) que governam a evolução dos processos. Para maior informação pode-se consultar os artigos $[3,20,17,16]$.

Para um enfoque diferente do modelo de aglomerados aleatórios pode ver-se o artigo de Welsh [21] que estuda o modelo desde o ponto de vista da teoria combinatória e para ver a relação do modelo com a entropia pode ver-se o artigo de Seppäläinen [19]. 


\section{Modelo de aglomerados aleatórios}

O modelo de aglomerados aleatórios que foi proposto por Fortuin e Kasteleyn [8] em 1972 é uma generalização do modelo da percolação de elos independentes e, em certo sentido, do modelo de Potts ferromagnético, sem campo magnético externo. Eles formularam uma família de medidas de probabilidade que depende de dois parâmetros. Esta família descreve um processo de percolação de elos dependentes ou interagentes. Portanto, o modelo de aglomerados é um processo sobre os elos de um grafo, com parâmetros $\left\{p_{e}\right\}$ e $q$, onde $p_{e}$ é a probabilidade do elo e do grafo estar aberto e $q$ é o parâmetro de aglomerados que introduz a dependência no modelo. Se a probabilidade $p_{e}$ para cada elo é independente do elo, então o modelo será dito homogêneo.

O modelo em sua curta existência teve muitas mudanças e generalizações depois da formulação de Fortuin e Kasteleyn. Ele voltou a ter importância e foi estudado mais profundamente nos trabalhos de Aizenman, Chayes L., Chayes J., Newman [2] em 1988 e Grimmett $[12,11]$ em 1995. Recentemente o modelo foi estudado em grafos abstratos nos trabalhos de Häggström [14] em 1998, Jonasson [16] em 1999 e Alexander [3] em 2000. Este último propõe uma forma de abordar o modelo de Potts com campo magnético externo usando o chamado modelo de aglomerados aleatórios assimétrico. 


\subsection{Definição do modelo.}

Consideremos o grafo finito $G_{\Lambda}, G_{\Lambda}=\left(\Lambda, E_{\Lambda}\right)$, onde $\Lambda$ é o conjunto de sítios e $E_{\Lambda}$ é o conjunto de elos que será expressado por

$$
E_{\Lambda}=\{e: e=<x, y>; x, y \in \Lambda \cup \partial \Lambda\}
$$

onde a notação $e=\langle x, y\rangle$ indica que os sítios $x$ e $y$ são adjacentes, isto é, $d(x, y)=1$, onde $d(.,$.$) é a distância euclidiana em G_{\Lambda}$, definida por $d(x, y)=\|x-y\| \quad \forall x . y \in \Lambda$. A cada elo de $E_{\Lambda}$ associamos uma função $\omega, \omega: E_{\Lambda} \rightarrow\{0,1\}$ de tal maneira que se $\omega(\epsilon)=1$ o elo $e$ será dito aberto e, se $\omega(e)=0$ o elo $e$ será dito fechado. Uma maneira simples de representar o conjunto de elos abertos e fechados é considerar o conjunto de zeros e uns para todos os elos de $E_{\Lambda}$. Este conjunto será definido como uma configuração, logo, o espaço de configurações do modelo de aglomerados é o conjunto $\Omega_{\Lambda}=\{0,1\}^{E_{\Lambda}}$, onde uma configuração é um vetor $\omega=\left\{\omega(e): \epsilon \in E_{\mathrm{.}}\right\}$.

Um conjunto de elos de $E_{\Lambda},\left\{e_{1}, e_{2}, \ldots, e_{n}\right\}, n \geq 1$, onde $e_{i}=<x_{i}, x_{i+1}>. i=$ $1,2 \ldots, n$, será dito um caminho se $x_{1}, x_{2}, \ldots, x_{n+1}$ forem distintos. Um caminho será dito aberto se todos seus elos estiveram abertos. Diremos que dois sítios $x$ e $y$ do grafo $G_{\Lambda}$, estão conectados (denotado por $x \leftrightarrow y$ ) se existir um caminho de elos abertos entre $x$ e $y$. Se definimos que cada sítio $x \in \Lambda$ está conectado consigo mesmo, vê-se que a conectividade é uma relação de equivalência e às classes de equivalência em que se dividem os sítios as chamaremos de aglomerados ou componentes conexas do grafo $G_{\Lambda}$.

Dado o grafo finito $G=(V, E)$ tal que $\Lambda \subseteq V \subset Z^{d}$ e $E_{\Lambda} \subseteq E \subset E^{d}$, então, o grafo $G_{\Lambda}$ é chamado subgrafo do grafo $G$. Denotamos o complemento do conjunto $\perp$ com respeito ao conjunto $V$ por $\Lambda^{c}$ e o definimos por $\Lambda^{c}=V \backslash \Lambda$. A fronteira do conjunto $\Lambda$ denotada por $\partial \Lambda$ é o conjunto de todos os sítios de $\Lambda^{c}$ que são adjacentes a algum sítio de $\Lambda$, ou seja

$$
\partial \Lambda=\left\{y \in Z^{d} \backslash \Lambda:<x, y>\in E^{d} \quad \forall x \in \Lambda\right\}
$$

Em vez de trabalhar com o vetor $\omega$ é conveniente trabalhar com o conjunto

$$
\eta(\omega)=\left\{e \in E_{\Lambda}: \omega(e)=1\right\}
$$


de elos abertos do grafo $G_{\Lambda}$. Com este conjunto podemos formar o subgrafo $(\Lambda, \eta(\omega))$ do grafo $G_{\Lambda}$, que esta formado por todos os sítios do grafo e por todos os elos dos aglomerados de elos abertos.

Definição 2.1. A medida de aglomerados aleatórios denotada por $\phi_{\Lambda,\left\{p_{e}\right\}, q}$ no grafo finito $G_{\Lambda}$ com parâmetros $\left\{p_{e}\right\}, p_{e} \in[0.1]$ para todo e e $q>0$, é uma medida de probabilidade definida sobre $\Omega_{\Lambda}$ tal que, a cada $\omega \in \Omega_{\Lambda}$ atribui a seguinte probabilidade

$$
\phi_{\Lambda,\left\{p_{e}\right\}, q}(\omega)=\frac{1}{Z_{\Lambda,\left\{p_{e}\right\}, q}}\left\{\prod_{e \in E_{\Lambda}} p_{e}{ }^{\omega(e)}\left(1-p_{e}\right)^{1-\omega(e)}\right\} q^{k(\omega)} ; \quad \omega \in \Omega_{\Lambda}
$$

onde $k(\omega)$ é o número de aglomerados do subgrafo $(\Lambda, \eta(\omega))$ do grafo $G_{\Lambda}$ que não tocam a fronteira de $\Lambda$ e $Z_{\Lambda,\left\{p_{e}\right\}, q}$ é a constante de normalização, dada por

$$
Z_{\Lambda,\left\{p_{e}\right\}, q}=\sum_{\omega \in \Omega_{\Lambda}}\left\{\prod_{e \in E_{\Lambda}} p_{e}{ }^{\omega(e)}\left(1-p_{e}\right)^{1-\omega(e)}\right\} q^{k(\omega)} .
$$

Escrevemos $\phi_{\Lambda,\left\{p_{e}\right\}, q}$, para enfatizar o papel dos parâmetros. O valor do parâmetro $q$ pode ser qualquer número real positivo. Será de muito interesse para nosso trabalho que $q$ seja inteiro, porque desta forma pode-se estabelecer uma correspondência entre o modelo de aglomerados e o modelo de Potts ferromagnético, que é muito estudado em Mecânica Estatística.

Note-se que se $q$ é igual a 1 esta medida,

$$
\phi_{\Lambda,\left\{p_{e}\right\}, 1}(\omega)=\prod_{e \in E_{\Lambda}} p_{e}^{\omega(e)}\left(1-p_{e}\right)^{1-\omega(e)}
$$

converte-se na medida produto, tratando-se assim do modelo de percolação de elos independentes. Neste caso o modelo de aglomerados é exatamente o mesmo modelo clássico da percolação de elos independentes em $G_{\Lambda}$. 


\subsection{Medida FKG.}

Faremos em seguida algumas definições que serão usadas para demonstrar propriedades do modelo de aglomerados aleatórios.

Rede sobre $\Omega_{\Lambda}$ (ver Birkhoff [4]).

Consideremos o conjunto $\Omega_{\Lambda}=\{0,1\}^{E_{\Lambda}}$. Denotamos por $\Omega_{\Lambda}^{2}$ o produto cartesiano de $\Omega_{\Lambda}$ consigo mesmo, então,

$\Omega_{\Lambda}^{2}=\left\{\left(w, w^{\prime}\right): w, w^{\prime} \in \Omega_{\Lambda}\right\}$.

Diremos que duas configurações são iguais, quando

$$
w=w^{\prime} \quad \Leftrightarrow \quad w(e)=w^{\prime}(e) \quad \forall e \in E_{\Lambda}
$$

Pode-se definir a relação binária $\leq$ sobre $\Omega_{\Lambda}$ da seguinte forma:

$$
w \leq w^{\prime} \quad \Leftrightarrow \quad w(e) \leq w^{\prime}(e) \quad \forall e \in E_{\Lambda}
$$

Que verifica as propriedades de um conjunto parcialmente ordenado:

(a) $w \leq w$

(b) $w \leq w^{\prime}$ e $w^{\prime} \leq w$, então $w=w^{\prime}$

(c) $w \leq w^{\prime}$ e $w^{\prime} \leq w^{\prime \prime}$, então $w \leq w^{\prime \prime}$.

Logo, o par $\left(\Omega_{\Lambda}, \leq\right)$ forma um conjunto parcialmente ordenado. Agora necessitamos definir o elemento máximo e o elemento mínimo para duas configurações quaisquer.

Dado $\Omega_{\Lambda}$ definimos o elemento máximo e mínimo de $\Omega_{\Lambda}$ por:

$$
\begin{aligned}
& \left(w \vee w^{\prime}\right)(e)=\max \left(w(e) ; w^{\prime}(e)\right) \quad \forall e \in E_{\Lambda}, e \forall w, w^{\prime} \in \Omega_{\Lambda} \\
& \left(w \wedge w^{\prime}\right)(e)=\min \left(w(e) ; w^{\prime}(e)\right) \quad \forall e \in E_{\Lambda}, e \forall w, w^{\prime} \in \Omega_{\Lambda}
\end{aligned}
$$

A relação de ordem $\leq$ sobre $\Omega_{\Lambda}$ formando um conjunto parcialmente ordenado com os elementos máximo e mínimo definem uma rede sobre $\Omega_{\Lambda}$ denotada por $\Gamma$. Diremos que uma rede é transitiva se $\forall x, y, z \in \Gamma$ cumpre-se

$$
x \vee(y \wedge z)=(x \wedge y) \vee(x \wedge z)
$$




\section{Função Crescente.}

Uma função $f: \Omega_{\Lambda} \rightarrow R$ é dita crescente se for crescente na ordem parcial acima, isto é,

$$
f(\omega) \leq f\left(\omega^{\prime}\right) \text { sempre que } \omega \leq \omega^{\prime},
$$

e será dita decrescente se $-f$ é crescente.

Um evento $A$ será dito crescente se sua função indicadora é crescente.

Definição 2.2. Dadas duas medidas de probabilidade $\phi$ e $\varphi$ definidas em $\Omega_{\Lambda}$, diremos que $\phi$ domina $\varphi$ no sentido FKG, denotado por $\phi \geq \varphi$. se para toda função $f: \Omega_{\Lambda} \rightarrow R$ crescente cumpre-se

$$
E_{\phi}(f) \geq E_{\varphi}(f)
$$

onde $E_{\phi}, E_{\varphi}$ são os valores médios sobre as medidas $\phi$ e $\varphi$ respectivamente.

O seguinte teorema dá uma condição para saber quando uma medida domina no sentido FKG a outra medida.

\section{Teorema 2.3. (Desigualdade de Holley)}

Seja uma rede finita transitiva $\Gamma$ e duas medidas de probabilidade $\mu_{1}$ e $\mu_{2}$ definidas sobre $\Omega_{\Lambda}$ tal que

$$
\mu_{1}\left(w_{1} \vee w_{2}\right) \mu_{2}\left(w_{1} \wedge w_{2}\right) \geq \mu_{1}\left(w_{1}\right) \mu_{2}\left(w_{2}\right) \quad \forall w_{1}, w_{2} \in \Omega_{\Lambda}
$$

Então, $\mu_{1}$ domina a $\mu_{2}$ no sentido FKG, isto é,

$$
E_{\mu_{1}}(f) \geq E_{\mu_{2}}(f)
$$

para toda função crescente $f: \Omega_{\Lambda} \rightarrow R$.

Prova. Pode ver-se no artigo original de Holley [15]. Outra prova mais moderna, usando a técnica de acoplamento pode ver-se em Grimmett [13]. 
Corolário 2.4. (Desigualdade FKG)

Seja uma rede finita transitiva $\Gamma$ e $\mu$ uma medida de probabilidade definida sobre $\Omega_{\Lambda}$ tal que

$$
\mu\left(\omega_{1} \vee \omega_{2}\right) \mu\left(\omega_{1} \wedge \omega_{2}\right) \geq \mu\left(\omega_{1}\right) \mu\left(\omega_{2}\right)
$$

para todo $\omega_{1}, \omega_{2} \in \Omega_{\Lambda}$, então,

$$
E_{\mu}[f g] \geq E_{\mu}[f] E_{\mu}[g]
$$

para todas as funções crescentes $f, g: \Omega_{\perp} \rightarrow R$.

\section{Prova.}

Dadas duas funções crescentes $f$ e $g$. Vamos supor sem perda de generalidade que $g$ é estritamente positiva, $\mu(g>0)>0$.

Definimos as medidas de probabilidade

$$
\mu_{1}(\omega)=\frac{g(\omega) \mu(\omega)}{\sum_{x \in \Omega_{\Lambda}} g(x) \mu(x)} \quad \text { e } \quad \mu_{2}(\omega)=\mu(\omega)
$$

em função da medida de probabilidade $\mu$. todas definidas sobre $\Omega_{\Lambda}$. Pela desigualdade de Holley, temos que se $\mu_{1}$ domina $\mu_{2}$ no sentido FKG, então, cumpre-se

$$
\sum_{x \in \Omega_{\Lambda}} f(x) \mu_{1}(x) \geq \sum_{x \in \Omega_{\Lambda}} f(x) \mu_{2}(x)
$$

do que obtemos

$$
\sum_{x \in \Omega_{\Lambda}} f(x) g(x) \mu(x) \geq \sum_{x \in \Omega_{\Lambda}} f(x) \mu(x) \sum_{x \in \Omega_{\Lambda}} g(x) \mu(x),
$$

o que prova o corolário. A prova original pode ver-se no artigo de Fortuin, Kasteleyn e Ginibre [9]

Definição 2.5. Uma medida definida sobre a rede $\Gamma$ é dita FKG se cumpre a desigualdade de FKG. 
Teorema 2.6. A medida de aglomerados aleatórios $\phi_{\Lambda,\left\{p_{e}\right\}, q}$, é FKG se $q \geq 1$.

Prova. Substituindo a medida $\phi_{\Lambda,\left\{p_{e}\right\}, q}$ na equação (2.6)

$$
o_{\Lambda,\left\{p_{e}\right\}, q}\left(\omega_{1} \vee \omega_{2}\right) \phi_{\Lambda,\left\{p_{e}\right\}, q}\left(\omega_{1} \wedge \omega_{2}\right) \geq \phi_{\Lambda,\left\{p_{e}\right\}, q}\left(\omega_{1}\right) \phi_{\Lambda,\left\{p_{e}\right\}, q}\left(\omega_{2}\right)
$$

daqui, depois de tomar logaritmos e simplificar obtemos

$$
k\left(\omega_{1} \vee \omega_{2}\right)+k\left(\omega_{1} \wedge \omega_{2}\right) \geq k\left(\omega_{1}\right)+k\left(\omega_{2}\right),
$$

façamos a troca de variáveis

$$
\eta=\omega_{1} \quad \text { e } \xi=\omega_{1} \wedge \omega_{2}
$$

Pela propriedade de rede temos que

$$
\omega_{2} \vee \xi=\omega_{2} \vee\left(\omega_{1} \wedge \omega_{2}\right)=\omega_{2}
$$

substituindo na equação (2.13)

$$
k\left(\eta \vee \omega_{2}\right)+k(\xi) \geq k(\eta)+k\left(\xi \vee \omega_{2}\right)
$$

daqui

$$
k\left(\eta \vee \omega_{2}\right)-k(\eta) \geq k\left(\xi \vee \omega_{2}\right)-k(\xi),
$$

finalmente (2.16) esta implicado pela afirmação de que a função

$$
h(\eta, \omega)=k(\eta \vee \omega)-k(\eta) ; \quad \eta, \omega \in \Omega_{\Lambda}
$$

é uma funçào não-decrescente, para todo $\omega$ fixo, de $\eta$. Isto pode ser provado pelo método da indução sobre o número de elos abertos na configuração $\omega$. Pode ver-se facilmente se $\omega$ tem só um elo aberto a função $h(\eta, \omega)$ é

$$
h(\eta, \omega)=\left\{\begin{array}{cc}
-1 & \text { se } \omega \text { pertence a } H(\eta) \\
0 & \text { caso contrário }
\end{array}\right.
$$

onde $H(\eta)$ é o evento: o elo aberto de $\omega$ conecta dois aglomerados distintos de $\eta$ ou conecta qualquer aglomerado com a fronteira. 
Disto temos que $h(\eta, \omega)$ é crescente. Agora podemos assumir que $h(\eta, \omega)$ é uma função crescente de $\eta$ para $\omega$ com menos de $n$ elos abertos. Suponhamos que $\omega$ tem exatamente $n$ elos abertos. Então, podemos escrever $\omega=\omega_{1}^{\prime} \vee \omega_{n-1}$, onde $\omega_{1}^{\prime}$ tem exatamente 1 elo aberto e $\omega_{n-1}$ tem exatamente $n-1$ elos abertos.

Usando a definição de $h(\eta, \omega)$ e somando e restando $k\left(\eta \vee \omega_{n-1}\right)$ temos

$$
h(\eta, \omega)=h\left(\omega_{n-1} \vee \eta, \omega_{1}^{\prime}\right)+h\left(\eta, \omega_{n-1}\right)
$$

Ou seja $h(\eta, \omega)$ é uma soma de duas funções crescentes e portanto é crescente. Isto prova o teorema.

\subsection{Desigualdades de comparação.}

O número de aglomerados aleatórios $k(\omega)$ tem as seguintes propriedades de monotonicidade:

(a) $k(\omega)$ é monótona decrescente em $\omega$, pois a adição de um elo pode só fazer decrescer o número de aglomerados.

(b) A função $k(\omega)+|\eta(\omega)|$ é monótona crescente em $\omega$. pois a adição de um elo pode decrescer $k(\omega)$ no máximo em um.

Estas propriedades nos permite estabelecer o seguinte

\section{Teorema 2.7. (Desigualdades de comparação)}

Sejam duas medidas $\phi_{\Lambda,\left\{p_{e}^{\prime}\right\}, q^{\prime}}$ e $\phi_{\Lambda,\left\{p_{e}\right\}, q}$, definidas em $\Omega_{\Lambda}$

(a)

$$
\operatorname{se}\left\{\begin{array}{c}
q^{\prime} \geq q, \quad q^{\prime} \geq 1 \quad e \\
p_{e}^{\prime} \leq p_{e} \quad \forall e
\end{array} \quad \text { então, } \quad \phi_{\Lambda,\left\{p_{e}^{\prime}\right\}, q^{\prime}} \leq \phi_{\Lambda,\left\{p_{e}\right\}, q}\right.
$$

(b)

$$
\text { se }\left\{\begin{array}{cl}
q^{\prime} \geq q, \quad q^{\prime} \geq 1 \quad e \\
\frac{p_{e}^{\prime}}{q^{\prime}\left(1-p_{e}^{\prime}\right)} \geq \frac{p_{e}}{q\left(1-p_{e}\right)} \quad \forall e
\end{array} \quad \text { então, } \quad \phi_{\Lambda \cdot\left\{p_{e}^{\prime}\right\}, q^{\prime}} \geq \phi_{\Lambda,\left\{p_{e}\right\}, q}\right.
$$


Prova. Para a prova usaremos as propriedades (a) e (b).

caso (a). Desde que $q^{\prime} \geq 1$. a medida $\phi_{\Lambda,\left\{p_{e}^{\prime}\right\}, q^{\prime}}$ é FKG.

Consideremos a medida

$$
\phi_{\Lambda,\left\{p_{e}\right\}, q}(\omega)=\frac{\phi_{\Lambda,\left\{p_{e}^{\prime}\right\}, q^{\prime}}(\omega) f(\omega)}{\sum_{x \in \Omega_{\Lambda}} \phi_{\Lambda,\left\{p_{e}^{\prime}\right\}, q^{\prime}}(x) f(x)} ; \quad \omega \in \Omega_{\Lambda}
$$

onde $f$ é escolhido da seguinte forma.

$$
f(\omega)=\left(\frac{q}{q^{\prime}}\right)^{k(\omega)} \prod_{e \in \Omega_{\Lambda}}\left(\frac{\frac{p_{e}}{1-p_{e}}}{\frac{p_{e}^{\prime}}{1-p_{e}^{\prime}}}\right)^{\omega(e)}
$$

das hipóteses obtemos

$$
\frac{q}{q^{\prime}} \leq 1 \quad e \quad \frac{p_{e}^{\prime}}{\left(1-p_{e}^{\prime}\right)} \leq \frac{p_{e}}{\left(1-p_{e}\right)}
$$

com isto vemos que $f$ é uma função crescente. Agora calculemos o valor médio com respeito a $\phi_{\Lambda,\left\{p_{e}\right\}, q}$ da função crescente $g$.

$$
E_{\phi_{\Lambda,\{p e}, q}(g)=\sum_{\omega \in \Omega_{\Lambda}} g(\omega) \phi_{\Lambda,\left\{p_{e}\right\}, q}(\omega)=\frac{\sum_{\omega \in \Omega_{\Lambda}} g(\omega) f(\omega) \phi_{\Lambda,\left\{p_{e}^{\prime}\right\}, q^{\prime}}(\omega)}{\sum_{x \in \Omega_{\Lambda}} \phi_{\Lambda,\left\{p_{e}^{\prime}\right\}, q^{\prime}}(x) f(x)}
$$

Usando a desigualdade de FKG, temos.

$$
E_{\phi_{\Lambda,\left\{p_{e}\right\}, q}}(g) \geq \sum_{\omega \in \Omega_{\Lambda}} g(\omega) \phi_{\Lambda,\left\{p_{e}^{\prime}\right\}, q^{\prime}}(\omega)=E_{\phi_{\Lambda,\left\{p_{e}^{\prime}\right\}, q^{\prime}}}(g)
$$

Por último, da definição de medida FKG terminamos a prova.

$$
\phi_{\Lambda,\left\{p_{e}\right\}, q} \geq \phi_{\Lambda,\left\{p_{e}^{\prime}\right\}, q^{\prime}}
$$

caso (b). Desde que $q^{\prime} \geq 1$. a medida $\phi_{\Lambda,\left\{p_{e}^{\prime}\right\}, q^{\prime}}$ é FKG.

Consideremos a medida

$$
\phi_{\Lambda,\left\{p_{e}\right\}, q}(\omega)=\frac{\phi_{\Lambda,\left\{p_{e}^{\prime}\right\}, q^{\prime}}(\omega) f(\omega)}{\sum_{x \in \Omega_{\Lambda}} \phi_{\Lambda,\left\{p_{e}^{\prime}\right\}, q^{\prime}}(x) f(x)} ; \quad \omega \in \Omega_{\Lambda}
$$

onde $f$ é escolhido da seguinte forma

$$
f(\omega)=\left(\frac{q}{q^{\prime}}\right)^{k(\omega)+|\eta(\omega)|} \prod_{e \in \Omega_{\Lambda}}\left(\frac{\frac{p_{e}}{q\left(1-p_{e}\right)}}{\frac{p_{e}^{\prime}}{q^{\prime}\left(1-p_{e}^{\prime}\right)}}\right)^{\omega(e)},
$$


das hipóteses obtemos

$$
\frac{q}{q^{\prime}} \leq 1 \quad \text { e } \quad \frac{p_{e}}{q\left(1-p_{e}\right)} \leq \frac{p_{e}^{\prime}}{q^{\prime}\left(1-p_{e}\right)}
$$

com isto vemos que $f$ é uma função decrescente. Agora calculemos o valor médio com respeito a $\phi_{\Lambda,\left\{p_{e}\right\}, q}$ da função decrescente $g$.

$$
E_{\phi_{\Lambda,\left\{p_{e}\right\}, q}}(g)=\sum_{\omega \in \Omega_{\Lambda}} g(\omega) \phi_{\Lambda,\left\{p_{e}\right\}, q}(\omega)=\frac{\sum_{\omega \in \Omega_{\Lambda}} g(\omega) f(\omega) \phi_{\Lambda,\left\{p_{e}^{\prime}\right\}, q^{\prime}}(\omega)}{\sum_{x \in \Omega_{\Lambda}} \phi_{\Lambda,\left\{p_{e}^{\prime}\right\}, q^{\prime}}(x) f(x)}
$$

Usando a desigualdade de FKG, temos.

$$
E_{\phi_{\Lambda,\left\{p_{e}\right\}, q}}(g) \geq \sum_{\omega \in \Omega_{\Lambda}} g(\omega) \phi_{\Lambda,\left\{p_{e}^{\prime}\right\}, q^{\prime}}(\omega)=E_{\phi_{\Lambda,\left\{p_{e}^{\prime}\right\}, q^{\prime}}}(g)
$$

multiplicando por -1

$$
E_{\phi_{\Lambda,\left\{p_{e}^{\prime}\right\}, q^{\prime}}}(-g) \geq E_{\phi_{\Lambda,\left\{p_{e}\right\}, q}}(-g)
$$

Por último, por ser $-g$ crescente, da definição de medida FKG terminamos a prova.

$$
\phi_{\Lambda,\left\{p_{e}^{\prime}\right\}, q^{\prime}} \geq \phi_{\Lambda,\left\{p_{e}\right\}, q}
$$

\subsection{Limite Termodinâmico}

Estamos interessados em estudar que acontece com as medidas de probabilidade do modelo de aglomerados aleatórios quando nosso grafo finito começa a crescer monotonicamente até se converter num grafo infinito, o chamado "limite termodinâmico". Um grafo infinito $G$ é o par $(V, E)$, onde $V$ é o conjunto infinito de sítios e $E$ é uma família infinita de pares não ordenados chamados elos. Se todos os sítios do grafo têm graus finitos, ou seja, o número de elos incidentes a qualquer sítio é finito, então, o grafo é dito localmente finito. Se todos os sítios têm o mesmo número de elos incidentes o grafo chama-se regular. O grafo que usaremos na dissertação é $\left(Z^{d}, E^{d}\right)$ que é um grafo localmente finito e regular, denotado por $L^{d}$, onde $Z^{d}$ é o conjunto de sítios dado por

$$
Z^{d}=\left\{x: x=\left(x_{1}, x_{2}, \ldots, x_{d}\right) ; x_{i} \in Z ; i=1,2, . ., . d\right\}
$$


e $E^{d}$ é o conjunto de elos com módulo euclideano igual a 1 (vizinhos próximos)

$$
E^{d}=\left\{e: e=<x, y>; x, y \in Z^{d}:\|x-y\|=1\right\} .
$$

Pelo resto da seção, consideraremos explicitamente apenas o caso de $L^{d}$. chamaremos atenção para o fato de que os resultados são validos, com as naturais adaptações, para o caso de um grafo infinito qualquer.

Consideremos o subconjunto $\Lambda \subset Z^{d}$, finito centrado na origem, dado por

$$
\Lambda=\left\{x \in Z^{d}:-L \leq x_{i} \leq L ; i=1,2, \cdots, d\right\} ; \quad L \in N
$$

Seu conjunto de elos é

$$
E_{\Lambda}=\{e: e=<x, y>; x, y \in \Lambda \cup \partial \Lambda:\|x-y\|=1\}
$$

Para uma configuração $\xi \in \Omega, \Omega=\{0,1\}^{E^{d}}$. definimos o conjunto de configurações

$$
\Omega_{\Lambda}^{\xi}=\left\{\omega \in \Omega: \omega(e)=\xi(e) \text { para } e \notin E_{\Lambda}\right\}
$$

A configuração $\xi$ introduz no modelo as condições de fronteira, importantes para a existência do limite termodinâmico. Para $\xi \in \Omega$ e valores de $\left\{p_{e}\right\}$ e $q$, satisfazendo $0 \leq p_{e} \leq 1$, para todo elo de $L^{d}$, e $q \geq 1$, definimos as medidas de aglomerados aleatórios $\phi_{\Lambda,\left\{p_{e}\right\}, q}^{\xi}$ sobre o conjunto $\Omega_{\Lambda}^{\xi}$.

$$
\phi_{\Lambda,\left\{p_{e}\right\}, q}^{\xi}(\omega)=\frac{1}{Z_{\Lambda,\left\{p_{e}\right\}, q}^{\xi}}\left\{\prod_{e \in E_{\Lambda}} p_{e}{ }^{\omega(e)}\left(1-p_{e}\right)^{1-\omega(e)}\right\} q^{k^{\xi}(\omega, \Lambda)} ; \quad \omega \in \Omega_{\Lambda}^{\xi}
$$

onde

$$
Z_{\Lambda,\left\{p_{e}\right\}, q}^{\xi}=\sum_{\omega \in \Omega_{\Lambda}^{\xi}}\left\{\prod_{e \in E_{\Lambda}} p_{e}{ }^{\omega(e)}\left(1-p_{e}\right)^{1-\omega(e)}\right\} q^{k^{\xi}(\omega, \Lambda)}
$$

é a constante de normalização, $k^{\xi}(\omega, \Lambda)$ é o número de aglomerados do grafo $\left(Z^{d}, \eta(\omega)\right)$ que intercepta $\Lambda$ na configuração que é igual a $\omega$ no conjunto $\Lambda$ e igual à configuração 
$\xi$ no conjunto $Z^{d} \backslash \Lambda$, sendo $\eta(\omega)$ o conjunto de elos abertos da configuração $\omega$.

Definição 2.8. A medida de probabilidade $\phi_{\left\{p_{e}\right\}, q}$ definida sobre o conjunto de configurações $\Omega=\{0,1\}^{E^{d}}$ associado ao grafo infinito $L^{d}$, é chamada a medida limite de aglomerados aleatórios com parâmetros $\left\{p_{e}\right\}$ para todo $e \in E^{d}$ e $q$ se existe uma condição de fronteira aleatória $\xi \in \Omega$ e uma seqüência crescente de subgrafos finitos $\left\{\left(\Lambda_{n}, E_{\Lambda_{n}}\right)\right\}_{n \geq 1}$, satisfazendo $\Lambda_{n} \rightarrow Z^{d}$ quando $n \rightarrow \infty$, tal que

$$
\phi_{\left\{p_{e}\right\}, q}=\lim _{\Lambda_{n} \nearrow Z^{d}} \phi_{\Lambda_{n},\left\{p_{e}\right\}, q}^{\xi}
$$

existe.

A medida $\phi_{\Lambda,\left\{p_{e}\right\}, q}^{\xi}$ de aglomerados aleatórios para $\xi(e)=1 \quad \forall e \in E^{d}$, tem um papel importante em nosso trabalho. A medida definida na equação (2.4) é igual à medida $\phi_{\Lambda,\left\{p_{e}\right\}, q}^{1}$ que é chamada medida de aglomerados aleatórios amarrada pelo fato que todos os elos fora da região finita $\Lambda$ estão abertos e portanto todos os sítios de $\Lambda^{c}$ estão conectados entre sí e logo com todos os sítios de $\Lambda$ que tocam a fronteira de $\Lambda$.

Note que $k^{1}(\omega, \Lambda)=k(\omega, \Lambda)+1$ (vide (2.4)) logo os fatores extras de $q$ no numerador e denominador, em relação às respectivas expressões da definição (2.4), se cancelam.

Existe muitos casos possíveis para as medidas $\phi_{\Lambda,\left\{p_{e}\right\}, q}^{\xi}$, dada uma configuração $\xi$. Por exemplo quando $\xi=0$, para todo elo, temos a medida de aglomerados aleatórios chamada livre.

Neste trabalho estaremos apenas interessados no caso $\xi(e)=1 \quad \forall e \in E^{d}$.

Portanto temos que $\phi_{\Lambda,\left\{p_{e}\right\}, q}^{1}=\phi_{\Lambda,\left\{p_{e}\right\}, q}$

O seguinte teorema estabelece a existência da medida de aglomerados no grafo infinito $L^{d}$, correspondente a este caso. Para o caso geral ver Grimmett [12] 
Teorema 2.9. (Limite Termodinâmico).

Dado o grafo $L^{d}$ e a medida $\phi_{\left\{p_{e}\right\}, q}$ definida sobre $\Omega$. O limite fraco

$$
\phi_{\left\{p_{e}\right\}, q}=\lim _{\Lambda \nearrow Z^{d}} \phi_{\Lambda,\left\{p_{e}\right\}, q}
$$

existe se $q \geq 1$.

Prova.

Consideremos duas caixas finitas $\Lambda$ e $\Lambda^{\prime}$ tal que $\Lambda \subset \Lambda^{\prime}$ e seja $p_{e}=p_{e}^{\prime} I_{\left\{e \in E_{\Lambda}\right\}}+1 I_{\left\{\epsilon \notin E_{\Lambda}\right\}}$, onde $\left\{p_{e}^{\prime}\right\}$ e $q$ são parâmetros da medida $\phi_{\Lambda^{\prime},\left\{p_{e}^{\prime}\right\}, q}$ e $\left\{p_{e}\right\}$ e $q$ são parâmetros da medida $\phi_{\Lambda,\left\{p_{e}\right\}, q}$.

Da desigualdade de comparação (a) (equação (2.19)), concluímos que

$$
\phi_{\Lambda^{\prime},\left\{p_{e}^{\prime}\right\}, q} \leq \phi_{\Lambda,\left\{p_{e}\right\}, q}
$$

Da monotonicidade de (2.38) concluímos que

$$
\lim _{\Lambda \nearrow Z^{d}} \phi_{\Lambda,\left\{p_{e}\right\}, q}=\phi_{\left\{p_{e}\right\}, q} \quad \text { existe }
$$

Observação. As desigualdades de comparação são válidas para $\phi_{\left\{p_{e}\right\}, q}$ pois são preservadas pelo limite.

\subsection{Transição de Fase}

Nesta seção consideraremos o caso homogêneo, ou seja $\left\{p_{e}\right\} \equiv p, \forall e \in L^{d}$. Consideremos o evento em $\Omega, \Omega=\{0,1\}^{E^{d}}$, de que um sítio qualquer $x$ do grafo esteja num aglomerado infinito de elos abertos, denotado por $\{x \leftrightarrow \infty\}$. Note que se trata de um evento crescente. Se a probabilidade do evento $\{x \leftrightarrow \infty\}$ sob $\phi_{p, q}$ é positiva dizemos que há percolação.

Definimos a probabilidade de percolação por

$$
\theta(p, q)=\phi_{p, q}(x \leftrightarrow \infty)
$$

Pela desigualdade de comparação $\theta(p, q)$ é uma função crescente em $p$ para $q \geq 1$ fixo. É claro que $\theta(0, q)=0$ e $\theta(1, q)=1$. 
Note-se que $\theta(p, q)$ é independente de $x \in Z^{d}$ porque o grafo $L^{d}$ é homogêneo e portanto $\phi_{p, q}(x \leftrightarrow \infty)$, invariante por translações.

Seja $p_{c}(q)=\sup \{p: \theta(p, q)=0\}$ a chamada probabilidade crítica para a percolação.

Teorema 2.10. Para $q \geq 1$,

(a) Se $d=1$, então, $p_{c}(q) \equiv 1$

(b) Se $d \geq 2$, então, $0<p_{c}(q)<1$

A parte (b) do teorema (2.10) estabelece a existência de transição de fase no modelo de aglomerados aleatórios em $L^{d}$ para $q \geq 1$ e $d \geq 2$.

No caso $q=1$, correspondente ao modelo de percolação independente, a probabilidade de percolação é denotada por $\theta(p)$. Neste caso temos que $p_{c}(1)=p_{c}$, cumprindo-se em $d \geq 2$,

$$
\theta(p)\left\{\begin{array}{l}
=0 \quad \text { se } p<p_{c} \\
>0 \quad \text { se } p>p_{c}
\end{array},\right.
$$

onde $p_{c} \in(0,1)$ é a probabilidade crítica.

A seguinte proposição que compara probabilidades críticas para diferentes modelos de aglomerados com diferentes parâmetros permitirá demonstrar o teorema (2.10).

Proposição 2.11. Se $q^{\prime} \geq q \geq 1$, então

$$
p_{c}(q) \leq p_{c}\left(q^{\prime}\right) \leq \frac{q^{\prime} p_{c}(q)}{q+\left(q^{\prime}-q\right) p_{c}(q)}
$$

Prova da proposição (2.11)

Primeiro provaremos

$$
p_{c}(q) \leq p_{c}\left(q^{\prime}\right)
$$


Da primeira desigualdade de comparação temos

$$
\text { se }\left\{\begin{array}{l}
q^{\prime} \geq q, \quad q^{\prime} \geq 1 \\
p^{\prime} \leq p
\end{array} \text { então, } \theta\left(p^{\prime}, q^{\prime}\right) \leq \theta(p, q)\right.
$$

Se $p \leq p_{c}(q)$, temos que $\theta\left(p^{\prime}, q^{\prime}\right) \leq \theta(p, q)=0$. Ademais

$$
p_{c}\left(q^{\prime}\right)=\sup \left\{p^{\prime}: \theta\left(p^{\prime}, q^{\prime}\right)=0\right\}
$$

Obtem-se que o conjunto $\left\{p^{\prime}<p ; p<p_{c}\right\} \subset\left\{p^{\prime}: \theta\left(p^{\prime}, q^{\prime}\right)=0\right\}$. Logo, $\sup \left\{p^{\prime}<p ; p<p_{c}\right\} \leq \sup \left\{p^{\prime}: \theta\left(p^{\prime}, q^{\prime}\right)=0\right\}$, mais ainda, $\sup \left\{p^{\prime}<p ; p<p_{c}\right\}=\sup \left\{p: p<p_{c}\right\}=p_{c}(q)$

Portanto, $p_{c}(q) \leq p_{c}\left(q^{\prime}\right)$

Isto prova a equação (2.41).

Agora provaremos

$$
p_{c}\left(q^{\prime}\right) \leq \frac{q^{\prime} p_{c}(q)}{q+\left(q^{\prime}-q\right) p_{c}(q)}
$$

Da segunda desigualdade de comparação temos

$$
\text { se }\left\{\begin{array}{l}
q^{\prime} \geq q, \quad q^{\prime} \geq 1 \\
\frac{p^{\prime}}{q^{\prime}\left(1-p^{\prime}\right)} \geq \frac{p}{q(1-p)}
\end{array} \text { então, } \theta\left(p^{\prime}, q^{\prime}\right) \geq \theta(p, q)\right.
$$

Se $p \geq p_{c}(q)$, temos que $\theta\left(p^{\prime}, q^{\prime}\right) \geq \theta(p, q)>0$. Ademais

$$
p_{c}\left(q^{\prime}\right)=\inf \left\{p^{\prime}: \theta\left(p^{\prime}, q^{\prime}\right)>0\right\}
$$

Obtem-se que o conjunto $\left\{\frac{p^{\prime}}{q^{\prime}\left(1-p^{\prime}\right)} \geq \frac{p}{q(1-p)} ; p>p_{c}\right\} \subset\left\{p^{\prime}: \theta\left(p^{\prime}, q^{\prime}\right)>0\right\}$. Logo,

$\left.\inf \left\{\frac{p^{\prime}}{q^{\prime}\left(1-p^{\prime}\right)} \geq \frac{p}{q(1-p)} ; p>p_{c}\right\} \geq \inf \left\{p^{\prime}: \theta\left(p^{\prime}, q^{\prime}\right)>0\right\}\right\}$, disto temos

$$
\inf \left\{\frac{p^{\prime}}{q^{\prime}\left(1-p^{\prime}\right)} \geq \frac{p}{q(1-p)} ; p>p_{c}\right\} \geq p_{c}\left(q^{\prime}\right)
$$

Observe-se que a função $f\left(p^{\prime}\right)=\frac{p^{\prime}}{\left(1-p^{\prime}\right)}$ é crescente e $p^{\prime}=f^{-1}(x)=\frac{x}{(1+x)}$

Consideremos a desigualdade $\frac{p^{\prime}}{q^{\prime}\left(1-p^{\prime}\right)} \geq \frac{p}{q(1-p)}$ que em termos da função $f$ é 
$\frac{p^{\prime}}{1-p^{\prime}}=f\left(p^{\prime}\right) \geq \frac{q^{\prime} p}{q(1-p)}$, tomando a função inversa temos $p^{\prime} \geq f^{-1}\left(\frac{p q^{\prime}}{q(1-p)}\right)=\frac{p q^{\prime}}{q(1-p)+p q^{\prime}}$. Então,

$\inf \left\{\frac{p^{\prime}}{q^{\prime}\left(1-p^{\prime}\right)} \geq \frac{p}{q(1-p)} ; p>p_{c}\right\}=\inf \left\{p^{\prime} \geq \frac{p q^{\prime}}{q(1-p)+p q^{\prime}} ; p>p_{c}\right\}$, portanto

$\inf \left\{p^{\prime} \geq \frac{p q^{\prime}}{q(1-p)+p q^{\prime}} ; p>p_{c}\right\}=\inf \left\{\frac{p q^{\prime}}{q(1-p)+p q^{\prime}} ; p>p_{c}\right\}$, do que obtemos

$\inf \left\{\frac{p^{\prime}}{q^{\prime}\left(1-p^{\prime}\right)} \geq \frac{p}{q(1-p)} ; p>p_{c}\right\}=\frac{q^{\prime} p_{c}(q)}{q\left(1-p_{c}(q)+q^{\prime} p_{c}(q)\right)}$

substituindo na equação (2.47) obtemos

$\frac{q^{\prime} p_{c}(q)}{q\left(1-p_{c}(q)+q^{\prime} p_{c}(q)\right)} \geq p_{c}\left(q^{\prime}\right)$ daqui obtemos

$$
p_{c}\left(q^{\prime}\right) \leq \frac{q^{\prime} p_{c}(q)}{q+\left(q^{\prime}-q\right) p_{c}(q)}
$$

Isto prova a equação (2.44)

As duas desigualdades estão provadas, portanto o teorema está provado.

Prova do teorema 2.10. Vamos supor conhecido o resultado para $q=1$, ver Grimmett [10] ou Fontes [7].

(a) Caso $d=1$. Pela proposição (2.11) temos:

$p_{c}(q) \geq p_{c}(1)=1 ; q \geq 1$. Portanto $p_{c}(q)=1$

(b) Caso $d \geq 2$. Pela proposição (2.11) temos:

$p_{c}(1) \leq p_{c}(q) \leq \frac{q p_{c}(1)}{1+(q-1) p_{c}(1)}$, como $0<p_{c}(1)<1$ temos que $0<\frac{q p_{c}(1)}{1+(q-1) p_{c}(1)}<1$.

Portanto concluímos que $0<p_{c}(q)<1$. Isto prova o teorema. 
CAPÍTUlO 3

\section{Modelo de Potts}

O modelo de Potts (ver Wu [22]), é um modelo em Mecânica Estatística Clássica que tem como objetivo descrever as propriedades de materiais magnéticos em equilíbrio térmico. Estamos interessados particularmente em saber se existe e sob que condições a magnetização espontânea. Veremos que a magnetização está diretamente relacionado com a probabilidade de percolação do modelo de aglomerados visto anteriormente. Neste capítulo faremos o estudo do modelo de Potts na rede definida sobre o grafo $L^{d}$.

\subsection{Definição do modelo.}

Nosso objeto de estudo são sistemas físicos com muitos constituintes, partículas com momentos magnéticos chamados spins, as quais estão localizados nos sítios do grafo $L^{d}$. A cada sítio $x \in Z^{d}$ associamos uma variável aleatória $\sigma(x)$ tomando valores no conjunto não vazio $S, S=\{1,2, \ldots q\}, q \geq 2, \sigma(x)$ é interpretado como o valor do spin no sítio $x$. O caso quando $q=2$ corresponde ao modelo de Ising. Uma configuração é uma função $\sigma: Z^{d} \rightarrow S$ que atribui a cada sítio $x \in Z^{d}$ um valor do spin $\sigma(x) \in S$. Em outras palavras as configurações são elementos do espaço produto $S^{Z^{d}}$ denotado por $\Sigma$. Logo $\Sigma=S^{Z^{d}}=\{1,2, \ldots, q\}^{Z^{d}}$.

Temos spins localizados no grafo $L^{d}$. Spins vizinhos próximos estão interagindo entre si. Este tipo de sistema é descrito por uma hamiltoniana, que mede a energia 
de interação dos componentes do sistema. Definir a hamiltoniana para um grafo infinito carece de sentido físico e traz complicações matemáticas insolúveis. Por isto. definiremos a hamiltoniana no grafo finito $G_{\Lambda}, G_{\Lambda}=\left(\Lambda, E_{\Lambda}\right)$, definido no capítulo anterior.

A hamiltoniana na região $\Lambda$ com a condição de fronteira $\xi$ é dada por

$$
H_{\Lambda}(\sigma \mid \xi)=-\sum_{\langle x . y\rangle ; x \in \Lambda, y \in \Lambda} J_{x y}(\delta(\sigma(x), \sigma(y))-1)-\sum_{\left\langle x, y>; x \in \Lambda, y \in \Lambda^{c}\right.} J_{x y}(\delta(\sigma(x), \xi(y))-1)
$$

onde $\sigma \in\{1,2, \ldots q\}^{\Lambda} ; \xi \in\{1,2, \ldots, q\}^{Z^{d} \backslash \Lambda} ; \sum_{<x, y>}$ denota soma sobre todos os elos $<x, y>\operatorname{com} x \in \Lambda, y \in \Lambda$ tais que $d(x, y)=\|x-y\|=1, J_{x y} \in R, J_{x y} \geq 0$, para ser modelo ferromagnético e $\delta(\sigma(x), \sigma(y))$ é o delta de Kronecker. A segunda soma à direita de (3.1) corresponde ao acoplamento com a condição de fronteira $\xi$. O caso $\left\{J_{x y}\right\}=J \geq 0 \quad \dashv x, y \in Z^{d}$ é dito homogêneo.

No Modelo de Potts existem pelo menos $(q+1)$ condições de fronteira. A condição de fronteira livre e as $q$ condições de fronteira de valores $\{1,2, \cdots, q\}$. No caso de fronteira livre temos que $J_{x y}=0$ quando $x \in \Lambda$ e $y \in \Lambda^{c}$, isto equivale a dizer que nào há spins fora da região $\Lambda$. Outro caso de interesse será quando $\xi(x)=s, \quad \forall x \in Z^{d} \backslash \Lambda$, onde $s$ é constante que pertence ao conjunto $\{1,2, \cdots, q\}$. Isto significa que todos os spins fora da região $\Lambda$ têm o mesmo valor.

No caso de fronteira livre a hamiltoniana não depende da condição de fronteira $\xi$, então temos

$$
H_{\Lambda}^{l}(\sigma \mid \xi):=H_{\Lambda}^{l}(\sigma)=-\sum_{<x, y>; x \in \Lambda, y \in \Lambda} J_{x y}(\delta(\sigma(x), \sigma(y))-1) \quad ; \quad \sigma \in \Sigma_{\Lambda}
$$

Como os valores dos spins são supostos aleatórios, consideramos convenientes medidas de probabilidade $\pi$ sobre o espaço $\Sigma$. Cada medida $\pi$ será dita um campo aleatório. Medidas de Gibbs, então, são campos aleatórios que descrevem sistemas físicos de spins quando estes estão em equilíbrio térmico.

Usaremos a seguinte notação para representar a família dos coeficientes de acopla- 
mento para o modelo de Potts.

$$
\mathcal{J}=\left\{J_{e}: e=<x, y>\forall x, y \in Z^{d}\right\}
$$

Definimos as medidas $\pi_{\Lambda}$ sobre o espaço de configurações $\Sigma_{\Lambda}, \Sigma_{\Lambda}=\{1,2, \ldots, q\}^{\Lambda}$, com a hamiltoniana dada por (3.1), como a distribuição de Gibbs com as condições de fronteira $\xi$ dada por

$$
\pi_{\Lambda, \beta, \mathcal{J}}^{\xi}(\sigma)=\frac{1}{Z_{\Lambda, \beta, \mathcal{J}}^{\xi}} \exp \left\{-\beta H_{\Lambda}(\sigma \mid \xi)\right\} \quad ; \quad \sigma \in \Sigma_{\Lambda}
$$

onde $Z_{\Lambda, \beta, \mathcal{J}}^{\xi}$ é a constante de normalização que é chamada função de partição. O parâmetro $\beta$ é chamada temperatura inversa e é definida como $\beta=\frac{1}{k_{B} T}$, sendo $k_{B}$ a constante de Boltzmann e $T$ a temperatura do meio. Por condição de normalização temos

$$
Z_{\Lambda, \beta, \mathcal{J}}^{\xi}=\sum_{\sigma \in \Omega_{\Lambda}} \exp \left\{-\beta H_{\Lambda}(\sigma \mid \xi)\right\}
$$

Com a medida dada por $(3,3)$ pode-se tomar valores médios de observáveis físicas.

Da mesma maneira como definiou-se a medida com condições de fronteira constante, pode-se definir a medida de Gibbs com condições de fronteira livres. A medida de Gibbs para este caso é dada por

$$
\pi_{\Lambda, \beta, \mathcal{J}}^{l}(\sigma)=\frac{1}{Z_{\Lambda, \beta, \mathcal{J}}^{l}} \exp \left\{-\beta H_{\Lambda}^{l}(\sigma)\right\} \quad ; \quad \sigma \in \Sigma_{\Lambda}
$$

$\operatorname{com} Z_{\Lambda, \beta, \mathcal{J}}^{l}$ como constante de normalização que é chamada também função de partição. Por condição de normalização temos

$$
Z_{\Lambda, \beta, \mathcal{J}}^{l}=\sum_{\sigma \in \Omega_{\Lambda}} \exp \left\{-\beta H_{\Lambda}^{l}(\sigma)\right\}
$$

Uma medida de Gibbs $\pi$ sobre o grafo infinito pode ser obtida como um limite fraco das medidas $\pi_{\Lambda}$ definida para uma seqüência $\left\{\left(\Lambda_{n}, E_{\Lambda_{n}}\right)\right\}_{n \geq 1}$ crescente de subgrafos finitos, satisfazendo que, $\Lambda_{n} \rightarrow Z^{d}$ quando $n \rightarrow \infty$. A medida limite é chamada limite termodinâmico. 
Um dos objetivos da Mecânica Estatística é entender a conexão entre as leis microscópicas (muitas variáveis) e leis macroscópicas (poucas variáveis), em particular uma pequena mudança dos parâmetros na lei microscópica pode ocasionar um efeito drástico nas leis macroscópicas. Este fenômeno é conhecido como transição de fase. A transição de fase pode ser detectada estudando o valor esperado (sob alguma medida de Gibbs apropriada) de um observável físico chamado parâmetro de ordem. Para nosso modelo usaremos a magnetização como parâmetro de ordem.

Agora apresentamos os resultados principais do modelo de Potts.

Definição 3.1. Consideremos o modelo de Potts com interação ferromagnética de vizinhos mais próximos sobre $Z^{d}$. Definimos a magnetização no ponto $x$ do volume finito como

$$
m_{\Lambda, x}(\beta, \mathcal{J}, q)=\frac{q}{q-1}\left\{\pi_{\Lambda, \beta, \mathcal{J}}^{s}(\sigma(x)=s)-q^{-1}\right\}
$$

onde $\sigma(x)$ é o spin no sítio $x \in \Lambda, s$ pode ser qualquer valor de $\{1,2, \ldots, q\}$.

Observação 3.1' Note-se que $m_{\Lambda . x}(\beta, \mathcal{J}, q)$ não depende de $s$

Observação 3.2' Podemos considerar também a magnetização com condições de fronteira livres dada por

$$
m_{\Lambda, x}^{l}(\beta, \mathcal{J}, q)=\frac{q}{q-1}\left\{\pi_{\Lambda, \beta, \mathcal{J}}^{l}(\sigma(x)=s)-q^{-1}\right\}
$$

note que, pela simetria de $H_{\Lambda}^{l}$ temos $\pi_{\Lambda, \beta, \mathcal{J}}^{l}(\sigma(x)=s)=q^{-1}$ para todo $s$. Portanto $m_{\Lambda, x}^{l}(\beta, \mathcal{J}, q) \equiv 0$.

Definição 3.2. A medida de probabilidade $\pi_{\beta, \mathcal{J}}^{s}$ definida sobre $\{1,2, \ldots, q\}^{Z^{d}}$ é chamada medida limite de Gibbs do modelo de Potts de $q$ estados sobre $Z^{d}$ com temperatura inversa $\beta$ e condições de fronteira $\xi=s \forall s \in\{1,2, \ldots, q\}$ constantes, se existir uma seqüência crescente de subgrafos finitos $\left\{\left(\Lambda_{n}, E_{\Lambda_{n}}\right)\right\}_{n \geq 1}$, satisfazendo $\Lambda_{n} \rightarrow Z^{d}$ quando $n \rightarrow \infty$ tal que 


$$
\pi_{\beta, \mathcal{J}}^{s}=\lim _{\Lambda_{n} \nearrow Z^{d}} \pi_{\Lambda_{n}, \beta, \mathcal{J}}^{s}
$$

existe.

Teorema 3.3 (Limite Termodinâmico). Para o modelo de Potts ferromagnético de vizinhos mais próximos com $q$ estados e condições de fronteira $s, s \in\{1,2 \ldots q\}$, definido sobre $Z^{d}, d \geq 1$, a temperatura inversa $\beta$, existe o limite termodinâmico

$$
\pi_{\beta, \mathcal{J}}^{s}=\lim _{\Lambda_{n} \nearrow Z^{d}} \pi_{\Lambda_{n}, \beta, \mathcal{J}}^{s}
$$

Em particular, existe a magnetização no volume infinito dado por

$$
m_{x}(\beta, \mathcal{J}, q)=\lim _{\Lambda_{n} \nearrow Z^{d}} m_{\Lambda_{n}, x}(\beta, \mathcal{J}, q)
$$

Este teorema será demonstrado abaixo usando a representação FK do modelo de Potts em termos do modelo de aglomerados aleatórios discutida a seguir.

\subsection{Representação FK}

Nesta seção, nós desenvolveremos a técnica que usaram Fortuin e Kasteleyn para obter a correspondência entre o modelo de aglomerados aleatórios e o modelo de Potts.

\section{Consideremos o fator de Gibbs}

$$
e^{-\beta H_{\Lambda}^{s}(\sigma)}, \quad \text { onde } \quad H_{\Lambda}^{s}(\sigma):=H_{\Lambda}(\sigma \mid \xi)
$$

com a hamiltoniana em condições de fronteira constantes $\xi(x)=s \quad \forall x \in Z^{d} \backslash .1$

$$
H_{\Lambda}^{s}(\sigma)=-\sum_{\langle x, y>; x \in \Lambda, y \in \Lambda} J_{x y}(\delta(\sigma(x), \sigma(y))-1)-\sum_{\langle x, y\rangle ; x \in \Lambda, y \in \Lambda^{c}} J_{x y}(\delta(\sigma(x), \xi(y))-1)
$$


Temos que

$$
\exp \left\{\beta J_{x y}(\delta(\sigma(x), \sigma(y))-1)\right\} \equiv\left\{\begin{array}{cc}
1 & \text { se } \sigma(x)=\sigma(y) \\
\exp \left\{-\beta J_{x y}\right\} & \text { se } \sigma(x) \neq \sigma(y)
\end{array}\right.
$$

disto temos

$$
\exp \left\{\beta J_{x y}(\delta(\sigma(x), \sigma(y))-1)\right\} \equiv\left(1-p_{x y}\right)+p_{x y} \delta(\sigma(x), \sigma(y))
$$

com

$$
p_{x y}=1-\exp \left\{-\beta J_{x y}\right\}
$$

ademais como $\beta \geq 0$ e $J_{x y} \geq 0$, então $p_{x y} \in[0,1]$, com isto tem-se

$$
e^{-\beta H_{\Lambda}^{s}(\sigma)}=\prod_{\left\langle x, y>: x \in \Lambda, y \in Z^{d}\right.}\left\{\left(1-p_{x y}\right)+p_{x y} \delta(\sigma(x), \sigma(y))\right\}
$$

O produto acima pode ser expandido numa soma de produtos indexado pelos subconjuntos $\Delta$ de elos $e, e=\langle x, y\rangle$, que aparecem no produto contribuindo com $p_{x y} \delta(\sigma(x), \sigma(y))$.

$$
\begin{gathered}
e^{-\beta H_{\Lambda}^{s}(\sigma)}=\sum_{\Delta \subset E_{\Lambda}} \prod_{e \in \Delta}\left\{p_{e} \delta(\sigma(x), \sigma(y))\right\} \prod_{e \in\left(E_{\Lambda} \backslash \Delta\right)}\left\{\left(1-p_{e}\right)\right\} \\
e^{-\beta H_{\Lambda}^{s}(\sigma)}=\sum_{\Delta \subset E_{\Lambda}} \prod_{e \in \Delta} p_{e} \prod_{e \in\left(E_{\Lambda} \backslash \Delta\right)}\left(1-p_{e}\right) \prod_{e \in \Delta} \delta_{e}(\sigma)
\end{gathered}
$$

onde

$$
\delta_{e}(\sigma)=\delta(\sigma(x), \sigma(y))= \begin{cases}1 & \text { se } \sigma(x)=\sigma(y) \\ 0 & \text { se } \sigma(x) \neq \sigma(y)\end{cases}
$$

é o delta de Kronecker.

$$
e^{-\beta H_{\Lambda}^{s}(\sigma)}=\sum_{\Delta \subset E_{\Lambda}} \prod_{e \in \Delta} p_{e} \prod_{e \in\left(E_{\Lambda} \backslash \Delta\right)}\left(1-p_{e}\right) \prod_{e \in \Delta} \delta_{e}(\sigma)
$$

Podemos ainda identificar subconjuntos de $E_{\Lambda}$ com configurações de elos abertos e fechados de $\Omega_{\Lambda}$, então

$$
e^{-\beta H_{\Lambda}^{s}(\sigma)}=\sum_{\omega \in \Omega_{\Lambda}} \prod_{e: \omega(e)=1} p_{e} \prod_{e: \omega(e)=0}\left(1-p_{e}\right) \prod_{e: \omega(e)=1} \delta_{e}(\sigma)
$$




$$
e^{-\beta H_{\Lambda}^{s}(\sigma)}=\sum_{\omega \in \Omega_{\Lambda}}\left\{\prod_{e \in E_{\Lambda}} p_{e}^{\omega(e)}\left(1-p_{e}\right)^{1-\omega(e)}\right\} \prod_{e: \omega(e)=1} \delta_{e}(\sigma)
$$

Agora a função de partição é dada por

$$
Z_{\Lambda, \beta, \mathcal{J}}^{s}=\sum_{\sigma \in \Sigma_{\Lambda}} \sum_{\omega \in \Omega_{\Lambda}}\left\{\prod_{e \in E_{\Lambda}} p_{e}{ }^{\omega(e)}\left(1-p_{e}\right)^{1-\omega(e)}\right\} \prod_{e: \omega(e)=1} \delta_{e}(\sigma)
$$

o produto interior não depende de $\sigma$, portanto

$$
Z_{\Lambda, \beta, \mathcal{J}}^{s}=\sum_{\omega \in \Omega_{\Lambda}}\left\{\prod_{e \in E_{\Lambda}} p_{e}{ }^{\omega(e)}\left(1-p_{e}\right)^{1-\omega(e)}\right\} \sum_{\sigma \in \Sigma_{\Lambda}} \prod_{e: \omega(e)=1} \delta_{e}(\sigma)
$$

O produto de deltas de Kronecker é 1 se somente se os spins que estão nos diferentes aglomerados disjuntos tomam o mesmo valor, portanto, temos

$$
\prod_{e: \omega(e)=1} \delta_{e}(\sigma)=\prod_{i=1}^{k^{\prime}} I_{\left\{\sigma \text { é constante em } A_{i}\right\}}
$$

onde $A_{1}, A_{2}, \ldots, A_{k^{\prime}}$ são aglomerados disjuntos de $(\Lambda, \eta(\omega))$. Logo temos

$$
Z_{\Lambda, \beta, \mathcal{J}}^{s}=\sum_{\omega \in \Omega_{\Lambda}}\left\{\prod_{e \in E_{\Lambda}} p_{e}{ }^{\omega(e)}\left(1-p_{e}\right)^{1-\omega(e)}\right\} \sum_{\sigma \in \Sigma_{\Lambda}} \prod_{i=1}^{k^{\prime}} I_{\left\{\sigma \text { é constante em } A_{i}\right\}}
$$

Seja $k, k \leq k^{\prime}$, o número de aglomerados disjuntos de $\omega$ que não tocam a fronteira de $\Lambda$ e $A_{1}, A_{2}, \ldots, A_{k}$ tais aglomerados. Desta forma $A_{k+1}, \ldots, A_{k^{\prime}}$ são aglomerados de $\omega$ que tocam a fronteira.

A segunda soma pode ser escrita como

$$
\sum_{\sigma_{1} \in \Sigma_{A_{1}}} \sum_{\sigma_{2} \in \Sigma_{A_{2}}} \cdots \cdots \sum_{\sigma_{k^{\prime}} \in \Sigma_{A_{k^{\prime}}}} \prod_{i=1}^{k^{\prime}} I_{\left\{\sigma \text { é constante em } A_{i}\right\}},
$$

onde $\sigma_{i}=\left.\sigma\right|_{A_{i}}$

A soma acima então fica

$$
\prod_{i=1}^{k}\left(\sum_{\sigma_{i} \in \Sigma_{A_{i}}} I_{\left\{\sigma \text { é constante em } A_{i}\right\}}\right) \cdot \prod_{i=k+1}^{k^{\prime}}\left(\sum_{\sigma_{i} \in \Sigma_{A_{i}}} I_{\left\{\sigma \text { é constante em } A_{i}\right\}}\right)
$$


Cada soma é igual a número de configurações constantes diferentes, isto é, igual a $q$ no primeiro produto acima e igual a 1 para o segundo produto acima. Desta forma o produto fica igual a $q^{k}$, então temos

$$
\sum_{\sigma \in \Sigma_{\Lambda}} \prod_{e: \omega(e)=1} \delta_{e}(\sigma)=q^{k(\omega)}
$$

onde $k$ é o número de aglomerados disjuntos de $(\Lambda, \eta(\omega))$ que não tocam a fronteira de $\Lambda$. Agora temos que a função de partição do modelo de Potts fica como

$$
Z_{\Lambda, \beta, \mathcal{J}}^{s}=\sum_{\omega \in \Omega_{\Lambda}} \prod_{e \in E_{\Lambda}}\left\{p_{e}{ }^{\omega(e)}\left(1-p_{e}\right)^{1-\omega(e)}\right\} q^{k(\omega)} \equiv Z_{\Lambda,\left\{p_{e}\right\}, q} .
$$

Note-se que esta função de partição coincide exatamente com a função de partição do modelo de aglomerados aleatórios. Isto significa que ambos modelos descrevem uma mesma realidade física o que estabelece uma equivalência entre os dois modelos.

Agora podemos obter uma expressão para a medida de Potts com condição de fronteira constante arbitraria.

$$
\pi_{\Lambda, \beta, \mathcal{J}}^{s}(\sigma)=\frac{1}{Z_{\Lambda, \beta, \mathcal{J}}^{s}} \exp \left\{-\beta H_{\Lambda}^{s}(\sigma)\right\} \quad ; \quad \sigma \in \Sigma_{\Lambda}
$$

Substituindo o fator de Gibbs dada pela equação (3.18) e usando a equação (3.20) temos

$$
\pi_{\Lambda, \beta, \mathcal{J}}^{s}(\sigma)=\frac{1}{Z_{\Lambda, \beta, \mathcal{J}}^{s}} \sum_{\omega \in \Omega_{\Lambda}}\left\{\prod_{e \in E_{\Lambda}} p_{e}{ }^{\omega(e)}\left(1-p_{e}\right)^{1-\omega(e)}\right\} \prod_{i=1}^{k^{\prime}(\omega)}\left\{I_{\left\{\sigma \text { é constante em } A_{i}\right\}}\right\}
$$

Multiplicando e dividindo o lado direita da equação (3.25) por $q^{k(\omega)}$ e usando o fato que $Z_{\Lambda, \beta, \mathcal{J}}^{s}=Z_{\Lambda,\left\{p_{e}\right\}, q}$ temos,

$\pi_{\Lambda, \beta, \mathcal{J}}^{s}(\sigma)=\frac{1}{Z_{\Lambda,\left\{p_{e}\right\}, q}} \sum_{\omega \in \Omega_{\Lambda}}\left\{\prod_{e \in E_{\Lambda}} p_{e}{ }^{\omega(e)}\left(1-p_{e}\right)^{1-\omega(e)}\right\} q^{k(\omega)} \prod_{i=1}^{k^{\prime}(\omega)}\left\{I_{\left\{\sigma \text { é constante em } A_{i}\right\}}\right\} q^{-k(\omega)}$

Introduzindo a constante de normalização dentro do primeiro produto e usando a definição do modelo de aglomerados teremos

$$
\pi_{\Lambda, \beta, \mathcal{J}}^{s}(\sigma)=\sum_{\omega \in \Omega_{\Lambda}} \phi_{\Lambda,\left\{p_{e}\right\}, q}(\omega) \prod_{i=1}^{k^{\prime}(\omega)}\left\{I_{\left\{\sigma \text { é constante em } A_{i}\right\}}\right\} q^{-k(\omega)}
$$


Definimos a $P_{\Lambda}^{s}(. \mid \omega)$ por

$$
P_{\Lambda}^{s}(\sigma \mid \omega)=q^{-k(\omega)} \prod_{i=1}^{k^{\prime}(\omega)}\left\{I_{\left\{\sigma \text { é constante em } A_{i}\right\}}\right\} ; \quad \sigma \in \Sigma_{\Lambda} .
$$

Sendo $A_{1}, A_{2}, \ldots ., A_{k}$ os aglomerados de $(\Lambda, \eta(\omega))$ que não tocam a fronteira de $\Lambda \mathrm{e}$ $A_{k+1}, \ldots . ., A_{k^{\prime}}$ os aglomerados que tocam a fronteira de $\Lambda$. Note que $k(\omega)$ é o mesmo número (aglomerados) que também usou-se para definir o modelo de aglomerados aleatórios.

$P_{.1}^{s}(. \mid \omega)$ é a probabilidade em $\Sigma_{\Lambda}$ sob a qual para cada aglomerados de $(\Lambda . \eta(\omega))$ que nào toca a fronteira é atribuído um spin constante, escolhido uniformemente ao acaso de $\{1,2, \ldots, q\}$, independentemente dos demais aglomerados e cada aglomerado que toca a fronteira com probabilidade um toma o valor do spin da fronteira. Portanto temos

$$
\pi_{\Lambda, \beta, \mathcal{J}}^{s}(\sigma)=\sum_{\omega \in \Omega_{\Lambda}} \phi_{\Lambda,\left\{p_{e}\right\}, q}(\omega) P_{\Lambda}^{s}(\sigma \mid \omega) ; \quad \sigma \in \Sigma_{\Lambda}
$$

Podemos ver que $P_{\Lambda}^{s}(. \mid \omega)$ é a medida condicional das configurações dos spins $\{\sigma\}$ dada as configurações dos elos $\{\omega\}$ com condições de fronteira constantes arbitrarias.

\subsection{Valores médios no modelo de Potts.}

Agora obteremos uma fórmula para o valor médio do modelo de Potts em função da medida condicional $P_{\Lambda}^{s}(. \mid \omega)$, onde $s$ indica as condições de fronteira constantes.

Seja $f: \Sigma_{\Lambda} \rightarrow R$ algum observável, o valor médio de $f(\sigma)$ define-se por

$$
\pi_{\Lambda, \beta, \mathcal{J}}^{s}(f):=\sum_{\sigma \in \Sigma_{\Lambda}} f(\sigma) \pi_{\Lambda, \beta, \mathcal{J}}^{s}(\sigma)=\sum_{\sigma \in \Sigma_{\Lambda}} \sum_{\omega \in \Omega_{\Lambda}} f(\sigma) \phi_{\Lambda,\left\{p_{e}\right\}, q} P_{\Lambda}^{s}(\sigma \mid \omega) .
$$


Ordenando temos

$$
\pi_{\Lambda, \beta, \mathcal{J}}^{s}(f)=\sum_{\omega \in \Omega_{\Lambda}} \phi_{\Lambda,\left\{p_{e}\right\}, q} E_{.1}^{s}(f \mid \omega) ; \quad \sigma \in \Sigma_{\Lambda}
$$

onde

$$
E_{\Lambda}^{s}(f \mid \omega):=\sum_{\sigma \in \Sigma_{\Lambda}} f(\sigma) P_{\Lambda}^{s}(\sigma \mid \omega) ; \quad \omega \in \Omega_{\Lambda}
$$

Provaremos um resultado muito importante que nos dá uma equivalência entre a magnetização no modelo de Potts e a percolação no modelo de aglomerados aleatórios.

Teorema 3.4. Para o Modelo de Potts no grafo finito $G_{\Lambda}$, a magnetização com condição de fronteira constante arbitraria $s, s \in\{1,2, . ., q\}$, satisfaz

$$
m_{\Lambda, x}(\beta, \mathcal{J}, q)=\frac{q}{q-1}\left\{\pi_{\Lambda, \beta, \mathcal{J}}^{s}(\sigma(x)=s)-q^{-1}\right\}=\phi_{\Lambda,\left\{p_{e}\right\}, q}(x \leftrightarrow \partial \Lambda)
$$

Prova. Pela representação FK:

$$
\begin{gathered}
\pi_{\Lambda, \beta, \mathcal{J}}^{s}(q \delta(\sigma(x), s)-1)=\sum_{\omega \in \Omega_{\Lambda}} \phi_{\Lambda,\left\{p_{e}\right\}, q} \sum_{\sigma \in \Sigma_{\Lambda}}(q \delta(\sigma(x), s)-1) P_{\Lambda}^{s}(\sigma \mid \omega) \\
\left.\pi_{\Lambda, \beta, \mathcal{J}}^{s}(q \delta(\sigma(x), s)-1)=\sum_{\omega \in \Omega_{\Lambda}} \phi_{\Lambda,\left\{p_{e}\right\}, q}\left\{q E_{\Lambda}^{s}(\delta(\sigma(x), s) \mid \omega)-1\right)\right\}
\end{gathered}
$$

Se na configuração $\omega$, o sítio $x$ não toca a fronteira, então $\sigma(x)$ é uniformemente distribuido em $\{1,2, . ., q\}$ sob $P_{\Lambda}^{s}(. \mid \omega), \log \circ, E_{\Lambda}^{s}(\delta(\sigma(x), s) \mid \omega)=$ $q^{-1}$, para todo $s$. Portanto a magnetização é zero.

Se na configuração $\omega$, o sítio $x$ toca a fronteira de $\Lambda(x \leftrightarrow \partial \Lambda)$, então $\left.\left\{q E_{\Lambda}^{s}(\delta(\sigma(x), s) \mid \omega)-1\right)\right\}=q-1$

Concluimos que o termo direito da equação (3.35) é igual a $(q-1) \phi_{\Lambda,\left\{p_{e}\right\}, q}(x \leftrightarrow \partial \Lambda)$. Portanto temos $m_{\Lambda}(\beta, \mathcal{J}, q)=\phi_{\Lambda,\left\{p_{e}\right\}, q}(x \leftrightarrow \partial \Lambda)$.

Isto prova o teorema.

Disto vemos que no limite termodinâmico a magnetização no modelo de Potts é simplesmente a probabilidade de percolação no modelo de aglomerados aleatórios. 
Teorema 3.5. Sobre $Z^{d}$, dados $\beta,\left\{p_{e}\right\}=\left\{1-e^{-\beta J_{e}}\right\}, e=<x, y>; \forall x, y \in Z^{d}$, e para qualquer condição de fronteira arbitraria $s \in\{1,2, \ldots, q\}$ cumpre-se

$$
m_{x}(\beta, \mathcal{J}, q)=\phi_{\left\{p_{e}\right\}, q}(x \leftrightarrow \infty)
$$

Prova. Usando o teorema (3.4) temos que

$$
m_{\Lambda, x}(\beta, \mathcal{J}, q)=\phi_{\Lambda,\left\{p_{e}\right\}, q}(x \leftrightarrow \partial \Lambda)
$$

Agora passando ao limite termodinâmico, teorema (3.3), obtemos a prova.

$$
m_{x}(\beta, \mathcal{J}, q)=\lim _{\Lambda \nearrow Z^{d}} \phi_{\Lambda,\left\{p_{e}\right\}, q}(x \leftrightarrow \partial \Lambda) .
$$

Veremos adiante o Lema (3.6) que mostra que o limite acima vale $\phi_{\left\{p_{e}\right\}, q}(x \leftrightarrow \infty)$

\subsection{Limite Termodinâmico para o Modelo de Potts.}

\section{Prova do teorema 3.3.}

Seja $f$ uma função cilíndrica e $A$ o seu suporte. Vamos mostrar a seguir que $\lim _{\Lambda \nearrow Z^{d}} \pi_{\Lambda, \beta, \mathcal{J}}^{s}[f]$ existe. Para todo $s, \beta, \mathcal{J}$ usando a resentação FK, para $A \subset \Lambda$, temos

$$
\pi_{\Lambda, \beta, \mathcal{J}}^{s}[f]=\sum_{\omega \in \Omega_{\Lambda}} \phi_{\Lambda,\left\{p_{e}\right\}, q}(\omega) E_{\Lambda}^{s}[f \mid \omega]
$$

onde $E_{\Lambda}^{s}[f \mid \omega]=\sum_{\sigma \in \Sigma_{\Lambda}} f(\sigma) P_{\Lambda}^{s}(\sigma \mid \omega) \operatorname{com} P_{\Lambda}^{s}(\sigma \mid \omega)$ definido por a equação (3.28). Consideremos a região $\Lambda^{\prime}$ satisfazendo $A \subset \Lambda^{\prime} \subset \Lambda$. Então, é claro que

$$
\left|E_{.1}^{s}[f \mid \omega]-E_{\Lambda^{\prime}}^{s}[f \mid \omega]\right|^{1} \leq 2\|f\|_{\infty} I_{\left\{\bigcup_{x \in A}\left\{e m \omega: x \leftrightarrow \partial \Lambda^{\prime}, x \nLeftarrow \partial \Delta\right\}\right.}
$$

portanto

$$
\pi_{\Lambda, \beta, \mathcal{J}}^{s}[f]=\sum_{\omega \in \Omega_{\Lambda}} \phi_{\Lambda,\left\{p_{e}\right\}, q}(\omega) E_{\Lambda^{\prime}}^{s}[f \mid \omega]+\varepsilon
$$

onde

$$
|\varepsilon| \leq 2\|f\|_{\infty} \sum_{x \in A} \phi_{\Lambda,\left\{p_{e}\right\}, q}\left(x \leftrightarrow \partial \Lambda^{\prime}, x \nLeftarrow \partial \Lambda\right) .
$$

\footnotetext{
${ }^{1}$ Aqui é bom esclarecer que para $\omega \in \Omega_{\Lambda^{\prime \prime}}$, com $\Lambda^{\prime} \subset \Lambda^{\prime \prime}$ temos: $E_{\Lambda^{\prime}}^{s}[f \mid \omega]:=E_{\Lambda^{\prime}}^{s}\left[f \mid \omega_{\Lambda^{\prime}}\right]$
} 
Como $E_{\Lambda^{\prime}}^{s}[f \mid \omega]$ é uma função cilíndrica de $\omega$ a somatória da equação (3.37) converge a

$$
\int \phi(d \omega) E_{\Lambda^{\prime}}^{s}[f \mid \omega] \text { quando } \Lambda \nearrow Z^{d}
$$

para $\omega \in \Sigma_{Z^{d}}$. Seja $P^{s}[. \mid \omega]$ a medida em $\Omega_{Z^{d}}$ que atribui spin uniforme em $\{1,2, \ldots, q\}$ a cada aglomerado finito de $\omega$, independentemente dos demais aglomerados, e atribui spin $s$ aos aglomerados infinitos de $\omega$.

É claro, então, que $E_{\Lambda^{\prime}}^{s}[f \mid \omega] \rightarrow E^{s}[f \mid \omega]$ quando $\Lambda^{\prime} \nearrow Z^{d}$ para todo $\omega \in \Sigma_{Z^{d}}$, onde

$$
E^{s}[f \mid \omega]=\int f(\sigma) d P^{s}(\sigma \mid \omega)
$$

Concluimos que a integral da equação (3.39) converge a $\int o(d \omega) E^{s}[f \mid \omega]$ quando $\Lambda^{\prime} \nearrow Z^{d}$, pelo teorema da convergencia dominada.

Vamos a considerar a expressão dereita da equação (3.38).

Para $x \in A$ :

$\phi_{\Lambda,\left\{p_{e}\right\}, q}\left(x \leftrightarrow \partial \Lambda^{\prime}, x \nLeftarrow \partial \partial \Lambda\right)=\phi_{\Lambda,\left\{p_{e}\right\}, q}\left(x \leftrightarrow \partial \Lambda^{\prime}\right)-\phi_{\Lambda,\left\{p_{e}\right\}, q}(x \leftrightarrow \partial \Lambda)$.

Como $\left\{x \leftrightarrow \partial \Lambda^{\prime}\right\}$ é cilíndrico:

$\phi_{\Lambda,\left\{p_{e}\right\}, q}\left(x \leftrightarrow \partial \Lambda^{\prime}\right) \rightarrow \phi_{\left\{p_{e}\right\}, q}\left(x \leftrightarrow \partial \Lambda^{\prime}\right)$ quando $\Lambda \nearrow Z^{d} \mathrm{e}$

$\phi_{\left\{p_{e}\right\}, q}\left(x \leftrightarrow \partial \Lambda^{\prime}\right) \rightarrow \phi_{\left\{p_{e}\right\}, q}(x \leftrightarrow \infty)$ quando $\Lambda^{\prime} \nearrow Z^{d}$.

Seja $\Lambda^{\prime \prime} \supset \Lambda$, então se cumpre

$$
\phi_{\Lambda,\left\{p_{e}\right\}, q}(x \leftrightarrow \partial \Lambda) \geq \phi_{\Lambda^{\prime \prime},\left\{p_{e}\right\}, q}(x \leftrightarrow \partial \Lambda),
$$

devido à monotonicidade da medida $\phi_{\Lambda^{\prime \prime},\left\{p_{e}\right\}, q}$, logo

$$
\phi_{\Lambda,\left\{p_{e}\right\}, q}(x \leftrightarrow \partial \Lambda) \geq \lim _{\Lambda^{\prime \prime} \nearrow Z^{d}} \phi_{\Lambda^{\prime \prime},\left\{p_{e}\right\}, q}(x \leftrightarrow \partial \Lambda)=\phi_{\left\{p_{e}\right\}, q}(x \leftrightarrow \partial \Lambda)
$$

Portanto

$$
\lim _{\Lambda \nearrow Z^{d}} \phi_{\Lambda,\left\{p_{e}\right\}, q}(x \leftrightarrow \partial \Lambda) \geq \phi_{\left\{p_{e}\right\}, q}(x \leftrightarrow \infty)
$$

Concluimos que $0 \leq \lim _{\Lambda^{\prime} \nearrow Z^{d}}\left\{\limsup _{\Lambda \nearrow Z^{d}} \varepsilon\right\} \leq 0$ e, logo, que

$$
\pi_{\Lambda, \beta, \mathcal{J}}^{s} \rightarrow \int \phi(d \omega) E^{s}[f \mid \omega] \text { quando } \Lambda \nearrow Z^{d}
$$

Isto prova o teorema. 


\section{Lema 3.6.}

$$
\lim _{\Lambda \nearrow Z^{d}} \phi_{\Lambda,\left\{p_{e}\right\}, q}(x \leftrightarrow \partial \Lambda)=\phi_{\left\{p_{e}\right\}, q}(x \leftrightarrow \infty) ; \forall x \in Z^{d}
$$

Prova. A equação (3.42) nos diz que

$$
\lim _{\Lambda \nearrow Z^{d}} \phi_{\Lambda,\left\{p_{e}\right\}, q}(x \leftrightarrow \partial \Lambda) \geq \phi_{\left\{p_{e}\right\}, q}(x \leftrightarrow \infty)
$$

Para obter a desigualdade recíproca. observe que para $\Lambda^{\prime} \subset \Lambda$, temos

$$
\phi_{\Lambda,\left\{p_{e}\right\}, q}(x \leftrightarrow \partial \Lambda) \leq \phi_{\Lambda,\left\{p_{e}\right\}, q}\left(x \leftrightarrow \partial \Lambda^{\prime}\right)
$$

Portanto

$$
\lim _{\Lambda \nearrow Z^{d}} \phi_{\Lambda,\left\{p_{e}\right\}, q}(x \leftrightarrow \partial \Lambda) \leq \lim _{\Lambda \nearrow Z^{d}} \phi_{\Lambda,\left\{p_{e}\right\}, q}\left(x \leftrightarrow \partial \Lambda^{\prime}\right)=\phi_{\left\{p_{e}\right\}, q}\left(x \leftrightarrow \partial \Lambda^{\prime}\right)
$$

pois $\left(\left\{x \leftrightarrow \partial \Lambda^{\prime}\right\}\right.$ é cilíndrico).

Como $\Lambda^{\prime}$ é arbitrario, temos que

$$
\lim _{\Lambda \nearrow Z^{d}} \phi_{\Lambda,\left\{p_{e}\right\}, q}(x \leftrightarrow \partial \Lambda) \leq \lim _{\Lambda^{\prime} \nearrow Z^{d}} \phi_{\left\{p_{e}\right\}, q}\left(x \leftrightarrow \partial \Lambda^{\prime}\right)=\phi_{\left\{p_{e}\right\}, q}(x \leftrightarrow \infty)
$$

Isto prova o lema.

\subsection{Transição de Fase no Modelo de Potts.}

Obteremos agora a transição de fase para o modelo de Potts usando o modelo de aglomerados aleatórios

Se $q=1$ temos que o modelo de aglomerados é o modelo de percolação de elos independentes e se $q^{\prime}=q$ o modelo de aglomerados $\operatorname{com}\left\{p_{e}\right\}=\left\{1-e^{-\beta J_{e}}\right\}$ é equivalente ao modelo de Potts não homogêneo com $q$ estados, constantes de acoplamento $\mathcal{J}$ e temperatura inversa $\beta$.

Vamos agora considerar o modelo homogêneo, isto é, $\left\{J_{e}\right\} \equiv J \quad \forall e \in E^{d}$. Seja

$$
\beta_{c}(d, q)=\sup \{\beta \geq 0: m(\beta, \mathcal{J}, q)=0\}
$$


a temperatura inversa crítica do modelo de Potts.

Pela proposição (2.11) tem-se

$$
p_{c} \leq p_{c}(q) \leq \frac{q p_{c}}{1+(q-1) p_{c}} ; \quad p_{c}(1):=p_{c}
$$

Da relaçào $p_{c}(q)=1-e^{-\beta_{c} J}$, obtem-se

$$
-\frac{1}{J} \ln \left(1-p_{c}\right) \leq \beta_{c} \leq-\frac{1}{J} \ln \left(1-\frac{q p_{c}}{1+(q-1) p_{c}}\right)
$$

Esta desigualdade nos dá cotas para a temperatura crítica.

Teorema 3.7. Para o modelo de Potts ferromagnético homogêneo de vizinhos mais próximos com $q$ estados e condições de fronteira positivas definido sobre $Z^{d}$. Cumpre-se:

(a) $\operatorname{Em} d=1, \beta_{c}(d, q)=\infty$

(b) $\operatorname{Em} d \geq 2, \beta_{c}(d, q) \in(0, \infty)$

Prova. É imediato da equação (3.45)

Em $d=1$. temos que $m(\beta, \mathcal{J}, q)=0 \quad \forall \beta<\infty, \mathcal{J}<\infty$.

Portanto $\{\beta \geq 0: m(\beta, \mathcal{J}, q)>0\}$ é o conjunto universal. Logo $\beta_{c}(d, q)=\infty$ Em $d \geq 2$, temos que $\beta_{c}=-\frac{1}{J} \ln \left(1-p_{c}\right)$. Agora como $0<p_{c}<1$ obtemos as seguintes desigualdades:

$-\ln \left(1-p_{c}\right)>0 \mathrm{e}-\ln \left(1-\frac{q p_{c}}{1+(q-1) p_{c}}\right)<\infty$.

Isto prova o teorema.

Observação. Pela monotonicidade de $m(\beta, \mathcal{J}, q)$ em $\beta$, temos que, em $d \geq 2$.

Se $\beta<3_{\varepsilon}$, então $m(\beta, \mathcal{J}, q)=0$

Se $\beta>3$. então $m(\beta, \mathcal{J}, q)>0$

Dizemos então que há transiçaõ de fase no modelo de Potts em $\beta=\beta_{c}$. 


\section{Modelo de Potts diluído}

Neste capítulo usaremos o modelo de aglomerados aleatórios para estudar algumas propriedades do modelo de Potts ferromagnético diluído (ver [1]). Particularmente estamos interessados em saber sob que condições ocorre a magnetização. Modelos de Potts desordenados são modelos de Potts não-homogêneos definidos sobre um grafo infinito $G, G(V, E)$, onde os coeficientes de acoplamento $\left\{J_{x y}\right\}_{<x, y>\in E}$ são uma família de variáveis aleatórias. Se esta família de variáveis aleatórias é não-negativa, então, o modelo será dito ferromagnético. Faremos o estudo deste modelo no grafo $L^{d}$.

\subsection{Definição do modelo}

Consideremos o modelo de Potts ferromagnético no grafo finito $\left(\Lambda, E_{\Lambda}\right)$, onde $\Lambda \subseteq V$, com diluição de elos. Este é um modelo de Potts com interação ferromagnética de pares vizinhos mais próximos. A hamiltoniana do modelo é dada por

$H_{\Lambda}(\sigma \mid \xi)=-\sum_{\langle x, y>; x \in \Lambda, y \in \Lambda} J_{x y}(\delta(\sigma(x), \sigma(y))-1)-\sum_{<x, y>; x \in \Lambda, y \in \Lambda^{c}} J_{x y}(\delta(\sigma(x), \xi(y))-1)$

onde $\sigma \in\{1,2, . ., q\}^{\Lambda} ; \xi \in\{1,2 . ., q\}^{Z^{d} \backslash \Lambda}$ e $\langle x, y\rangle$ indica um par genérico de sítios $x, y$ vizinhos mais próximos em $L^{d}, \sigma(x)$ é o valor do spin no sítio $x$. 
O modelo será chamado de elos diluídos se os acoplamentos

$$
\mathcal{J}:=\left\{J_{e}: e=<x, y>\in E^{d}\right\}
$$

são variáveis aleatórias não-negativas independentes e identicamente distribuídas. Mais especificamente consideraremos o caso em que $\left\{J_{e}\right\}_{e \in E^{d}}$ é um conjunto de zeros e uns, onde $J_{e}=0$ indica que o elo $e$ está diluído e $J_{e}=1$ indica que o elo $e$ está não-diluído. Associemos ao modelo diluído o seguinte espaço de probabilidade $\left(\Omega_{\mathcal{J}}, \Delta, \mathbb{P}\right)$. Como espaço amostral tomamos o conjunto $\Omega_{\mathcal{J}}, \Omega_{\mathcal{J}}=\{0,1\}^{E^{d}}$, onde uma configuração é $\mathcal{J}=\left\{J_{e}: e \in E^{d}\right\}$. A $\sigma$-álgebra é a usual, denotada por $\Delta$, gerada pelos eventos cilíndricos, isto é, aqueles que dependem de elos em subconjuntos finitos de $E^{d}$ apenas. Finalmente. tomamos a medida produto em $\Omega_{\mathcal{J}}$ dada por

$$
\mathbb{P}=\prod_{e \in E} \mathbb{P}_{e}
$$

onde $\mathbb{P}_{e}$ é a medida de Bernoulli definida sobre $\{0,1\}$ dada por

$$
\mathbb{P}_{e}\left(J_{e}=1\right)=1-\mathbb{P}_{e}\left(J_{e}=0\right)=p^{\prime} ; \quad e=<x, y>; \forall x, y \in Z^{d},
$$

com $p^{\prime} \in[0,1]$. A esperança com respeito a esta probabilidade será denotada por $\mathbb{E}$.

Dada uma realização do processo $\mathcal{J}$ sob temperatura inversa $\beta$, podemos introduzir as medidas de Gibbs $\pi_{3, \mathcal{J}}^{s}$ com condições de fronteira constantes $s \in\{1,2, . ., q\}$. A magnetização no ponto $x \in Z^{d}$ é dada por

$$
m_{x}(\beta, \mathcal{J}, q)=\frac{q}{q-1}\left\{\pi_{\beta, \mathcal{J}}^{s}(\sigma(x)=s)-q^{-1}\right\}=\phi_{\left\{p_{e}\right\}, q}(x \leftrightarrow \infty),
$$

que é uma função de $\mathcal{J}$ e portanto uma variável aleatória (não-trivial) sob $\mathbb{P}$. $\left\{p_{e}\right\}$ é a família das probabilidades dos elos estarem abertos dada uma realização de $\mathcal{J}$, onde $p_{e}=1-e^{-\beta J_{e}}$. Note que $m_{x}(\beta, \mathcal{J}, q)$ não depende de $s$.

Definamos a magnetização média $M(\beta, q)=\mathbb{E}\left(m_{x}(\beta, \mathcal{J}, q)\right)$, onde $\mathbb{E}$ é a esperança sob $\mathbb{P}$. Esta não é aleatória. Então temos, pela representação $\mathrm{FK}$,

$$
M(\beta, q)=\mathbb{E}\left\{\phi_{\left\{p_{e}\right\}, q}(x \leftrightarrow \infty)\right\},
$$

onde $\phi_{\left\{p_{e}\right\}, q}(x \leftrightarrow \infty)$ é a probabilidade de percolação do modelo de aglomerados aleatórios com a probabilidade do elo $e$ estar aberto dado por $p_{e}=1-e^{-\beta J_{e}}$. Note que $M(\beta, q)$ que não depende de $x$, pela invariância por translações de $\mathbb{P}$. 


\subsection{Resultados principais.}

Definimos a seguir um parâmetro crítico para a magnetização no modelo de Potts diluído.

$\operatorname{Seja} \beta_{c}(\mathcal{J})=\sup \left\{\beta \geq 0: m_{x}(\beta, \mathcal{J}, q)=0 \quad \forall x \in Z^{d}\right\}$

Proposição 4.1. Existe uma constante $\beta^{*} \in[0, \infty]$ tal que

$$
\beta_{c}(\mathcal{J})=\beta^{*} \text { quase-certamente. }
$$

Prova. Para $\beta \geq 0$, seja $A_{\beta}$ o evento

$$
A_{\beta}=\left\{\mathcal{J}: m_{x}(\beta, \mathcal{J}, q)=0 \quad \forall x \in Z^{d}\right\}=\left\{\mathcal{J}\left(p_{e}\right): \phi_{\left\{p_{e}\right\}, q}(x \leftrightarrow \infty)=0 \quad \forall x \in Z^{d}\right\}
$$

Então $A_{\beta}$ é invariante por translações de $\mathcal{J}$ para todo $\beta \geq 0$

Pela ergodicidade por translações de $\mathbb{P}$, por uma conhecida Lei $0-1, A_{\beta}$ é trivial para todo $\beta$.

Pelas desigualdades de comparação, $\mathbb{P}\left(A_{\beta}\right)$ é não crescente em $\beta$.

Seja $\beta^{*}=\sup \left\{\beta \geq 0: \mathbb{P}\left(A_{\beta}\right)=1\right\}(\sup (\phi)=0)$.

Temos então $\beta_{c}(\mathcal{J})=\beta^{*}$ quase-certamente

Agora já podemos estabelecer o seguinte resultado.

Teorema 4.2. Consideremos o modelo de Potts com $q$ estados, $q \geq 2$, diluído em $Z^{d}$ a temperatura inversa $\beta$, com distribuição $\mathbb{P}$ para os acoplamentos $\mathcal{J}$ e seja $p_{c}$ a probabilidade crítica de percolação de elos independentes em $Z^{d}$. Então cumpre-se

(a) $\operatorname{Em} d=1, \beta^{*}=\infty$, isto é, todos os sítios estão desmagnetizados para quase todo $\mathcal{J}$ e $\beta<\infty$.

(b) Em $d \geq 2$, temos:

(b1) Se $p^{\prime} \leq p_{c}$, então $\beta^{*}=\infty$.

(b2) Se $p^{\prime}>p_{c}$, então $0<\beta^{*}<\infty$.

Prova. Para provar o teorema, usaremos as desigualdades de comparação para o modelo de aglomerados aleatórios. Dada uma realização $\left\{p_{e}\right\}$, cumpre-se no sentido 
FKG a seguinte desigualdade

$$
\phi_{\Lambda, h\left(\left\{p_{e}\right\}\right), 1} \leq \phi_{\Lambda,\left\{p_{e}\right\}, q} \leq \phi_{\Lambda,\left\{p_{e}\right\}, 1} \quad \text { quando } \quad h\left(p_{e}\right)=\frac{p_{e}}{p_{e}+q\left(1-p_{e}\right)} .
$$

Todas as medidas de probabilidade são aleatórias porque dependem do parâmetro $p_{e}=1-e^{-\beta J_{e}}$. No limite termodinâmico temos

$$
\phi_{h\left(\left\{p_{e}\right\}\right), 1} \leq \phi_{\left\{p_{e}\right\}, q} \leq \phi_{\left\{p_{e}\right\}, 1}
$$

Sejam as constantes:

$$
p_{1}=\mathbb{E}\left(1-e^{-\beta J_{e}}\right) \quad p_{2}=\mathbb{E}\left(\frac{1-e^{-\beta J_{e}}}{1+(q-1) e^{-\beta J_{e}}}\right) .
$$

Logo temos que $p_{1}=\mathbb{E}\left(p_{e}\right)$ e $p_{2}=\mathbb{E}\left(h\left(p_{e}\right)\right)$.

Definamos as seguintes medidas determinísticas

$$
M(\beta, q)=\mathbb{E}\left(\phi_{\left\{p_{e}\right\}, q}\right), \quad \Phi_{1}=\mathbb{E}\left(\phi_{\left\{p_{e}\right\}, 1}\right) \quad \text { e } \quad \Phi_{2}=\mathbb{E}\left(\phi_{h\left(\left\{p_{e}\right\}\right), 1}\right)
$$

Note-se que $\Phi_{1}=\phi_{p_{1}, 1}$ e $\Phi_{2}=\phi_{p_{2}, 1}$ são modelos de percolação independentes com parâmetros $p_{1}$ e $p_{2}$ respectivamente. Tomando valores médios à equação (4.6), obtemos

$$
\theta\left(p_{2}\right) \leq M(3 . q) \leq \theta\left(p_{1}\right)
$$

onde $\theta(p)=\phi_{p, 1}(x \leftrightarrow \infty)$

Em $d=1, \theta(p)=0$ para todo $p<1$.

Portanto de (4.9) $M(\beta, q)=0 \quad \forall \beta<\infty$, pois $p_{1}<1$.

Portanto $m_{x}(\beta, \mathcal{J}, q)=0$ quase certamente para todo $x \in Z^{d}$.

Portanto $\beta^{*}=\infty$.

Em $d \geq 2$, e $p^{\prime} \leq p_{c}$, temos $p_{1}=\mathbb{E}\left(1-e^{-\beta J_{e}}\right)=\mathbb{P}_{e}\left(J_{e}=1\right)\left(1-e^{-\beta}\right)=$ $p^{\prime}\left(1-e^{-\beta}\right)<p^{\prime}$, então $p_{1}<p_{c}$, para todo $3<\infty$

Portanto $\theta\left(p_{1}\right)=0$. Então, de (4.9), $M(\beta . q)=0 \quad \forall \beta<\infty$.

Portanto $m_{x}(\beta, \mathcal{J}, q)=0$ quase certamente para todo $x \in Z^{d}$.

Portanto $\beta^{*}=\infty$.

Em $d \geq 2$, e $p^{\prime}>p_{c}$.

Se $p_{1}<p_{c} \Leftrightarrow \beta<\ln \left(\frac{p^{\prime}}{p^{\prime}-p_{c}}\right)$, entao, $\theta\left(p_{1}\right)=0$. Portanto $M(\beta, q)=0$. 
Portanto $m_{x}(\beta . \mathcal{J}, q)=0$ quase certamente para todo $x \in Z^{d}$ se $\beta<\ln \left(\frac{p^{\prime}}{p^{\prime}-p_{c}}\right)$

Portanto $\beta^{*} \geq \ln \left(\frac{p^{\prime}}{p^{\prime}-p_{c}}\right)>0$.

Se $p_{2}>p_{c} \Leftrightarrow \beta>\ln \left(\frac{p^{\prime}+(q-1) p_{c}}{p^{\prime}-p_{c}}\right)$, então

$\theta\left(p_{2}\right)>0$. Portanto $M(\beta, q)>0$ e $\mathbb{P}\left(m_{x}(\beta, \mathcal{J}, q)>0\right)>0$ para todo $x \in Z^{d}$.

Portanto $\beta^{*} \leq \ln \left(\frac{p^{\prime}+(q-1) p_{c}}{p^{\prime}-p_{c}}\right)<\infty$.

Isto prova o teorema.

Teorema 4.3. Em $d \geq 2, p^{\prime}>p_{c}$ e $\beta>\beta^{*}$ cumpre-se:

(a) Todo sítio $x$ num aglomerado finito de elos não diluidos está desmagnetizado para toda realização de $\mathcal{J}$.

(b) Para quase todo $\mathcal{J}$, todo sítio $x$ pertencente a um aglomerado infinito de elos não diluídos está magnetizado, isto é, satisfaz, $m_{x}(\beta, \mathcal{J}, q)>0$.

\section{Prova.}

(a) É trivial, já que nos aglomerados finitos temos modelos de Potts em volume finito com condições de fronteira livres, logo, para cada sítio de qualquer aglomerado finito a magnetização é zero.(vide teorema (3.4))

(b) Se $\beta>\beta^{*}$. então, quase certamente há pelo menos um sítio magnétizado, isto é, satifazendo $m_{x}(\beta, \mathcal{J}, q)>0$. x está num aglomerado infinito (denotado por $C$ ), pois caso contrário, $m_{x}(\beta, \mathcal{J}, q)=0$ (pelo raciocinio da parte (a) acima). Seja y um vizinho mais próximo de $x, y \in C$ tal que o elo $\langle x, y\rangle$ está aberto. Então

$$
\phi_{\left\{p_{e}\right\}, q}(y \leftrightarrow \infty) \geq \phi_{\left\{p_{e}\right\}, q}(y \leftrightarrow x, x \leftrightarrow \infty) \geq \phi_{\left\{p_{e}\right\}, q}(y \leftrightarrow x) . \phi_{\left\{p_{e}\right\}, q}(x \leftrightarrow \infty)
$$

A segunda desigualdede é obtida usando o teorema de FKG; o segundo termo do produto final é positivo pela hipóteses, pois $m_{x}(\beta, \mathcal{J}, q)=\phi_{\left\{p_{e}\right\}, q}(x \leftrightarrow \infty)>0$. O primeiro termo é positivo

$$
\dot{\phi}_{\left\{\left\{p_{e}\right\}, q\right\}}(y \leftrightarrow x) \geq \phi_{\left\{\left(p_{<x, y>}>1\right) ; p_{e} \equiv 0, e \neq<x, y>, q\right\}}(y \leftrightarrow x)>0
$$

pois a medida de probabilidade do evento: o elo $e=\langle x, y\rangle$ estar aberto e todos os demais elos estarem fechados, é positiva para todo $x, y$ vizinhos próximos. Ademais o evento: o elo $\epsilon=\langle x, y\rangle$ estar aberto e todos os demais elos estarem fechados, esta contenido no evento: o elos $e=\langle x, y\rangle \forall x, y$ é aberto ou fechado arbitrariamente. 
Portanto $m_{y}(\beta, \mathcal{J}, q)>0$.

Concluimos que $m_{y}(\beta, \mathcal{J}, q)>0$ para todo $y \in C$. Como $C$ é quase certamente único, o teorema está demonstrado.

\section{Observação.}

$\mathrm{Na}$ demonstração acima, usamos o fato de que no modelo de percolação de elos independentes em $Z^{d}$ há um único aglomerado infinito quase certamente na fase supercrítica. Isto é uma característica de $Z^{d}$. Em outros grafos homogêneos, pode ocorrer de haver mais que um aglomerado infinito, como por exemplo na árvore de Cayley, em que há infinitos.

Todos os resultados desta dissertação são validos nestes tipos de grafos, por essencialmente os mesmos argumentos, a não ser o teorema (4.3), que também é válido com os mesmos argumentos a não ser que não podemos usar a unicidade do aglomerado infinito. Podemos neste caso nos valer de um resultado recente (ver [17]) que estabelece a indistinguibilidade (probabilística) dos aglomerados infinitos quando há mais de um. 


\section{Referências Bibliográficas}

[1] M. Aizenman, J. Chayes, L. Chayes e M. Newman, The phase boundary in dilute Ising and Potts ferromagnets, J. Phys. A 20 (1987), no. 5, L313-L318.

[2] _ Discontinuity of the magnetization in one-dimensional $1 /|x-y|^{2}$ Ising and Potts models, J. Statist. Phys. 50 (1988), no. 1-2, 1-40.

[3] K. Alexander, The asymmetric random cluster model and q-states Potts model, preprint, 2000.

[4] G. Birkhoff, Lattices Theory, 3 ed., vol. XXV, American Mathematical Society, 1973.

[5] S. Bradbent e J. Hammersley, Percolation processes I. Crystal and mazes, Proceeding of the Cambridge Philosofical Society 53 (1957), 629-641.

[6] R. Ellis, Entropy, Large Desviations and Statistical Mechanics, Springer, New York, 1985.

[7] L. Fontes, Notas em percolação, Instituito de Matemática Pura e Aplicada, 1996.

[8] C. Fortuin e P. Kasteleyn, On the random cluster model I, Physica 57 (1972), $536-564$.

[9] C. Fortuin, P. Kasteleyn e J. Ginibre, Correlation inequalities on some partially ordered sets, Commun. Math. Phys. 22 (1971), 89-103. 
[10] G. Grimmett, Percolation, Springer, 1989.

[11] _ Percolative problems. In probability an Phase Transition. 69-86, Kluwer, Dordrecht, 1995, pp. 69-86.

[12] _ The stochastic random-cluster processes and the uniqueness of randomcluster measures, Annal of Probability 23 (1995), no. 4, 1461-1510.

[13] _ Percolation and disordered systems, Lecture notes in Mathematics. vol. 1665, Springer Berlin, 1997, pp. 153-300.

[14] O. Häggström, Random cluster representation in the study of phase transition, .Markov Processes Relat. Fields 4 (1998), 275-321.

[15] R. Holley, Remarks on the FKG inequalities, Commun. Math. Phys. 36 (1974), $227-231$.

[16] J. Jonasson, The random cluster model on genaral graph and the phase transition characterization of nonamenability, preprint, 1999.

[17] R. Lyons e O. Schramm, Indistinguishability of percolation clusters, preprint, 1999.

[18] D. Ruelle, Statistical Mechanics. Rigorous Results, Benjamin, New York, 1969.

[19] T. Seppäläinen, Entropy for translation-invariant random cluster measures. $A n$ nal of Probability 26 (1998), no. 3, 1139-1178.

[20] R. Shonmann, Stability of infinite cluster in supercritical percolation, Probab. Theory Relat. Fields 113 (1999), 287-300.

[21] D. Welsh, The random cluster process, Discrete mathematics 136 (1994), 373390.

[22] F. Wu, The Potts model, Rev. Mod. Phys. 54 (1982), 235-268. 